\title{
Expression of Castanea crenata Allene Oxide Synthase in Arabidopsis Improves the Defense to Phytophthora cinnamomi
}

\author{
Susana Serrazina ${ }^{1 *}$, Helena Machado ${ }^{2}$, Rita Lourenço Costa ${ }^{2,3}$, Paula Duque ${ }^{4}$ and \\ Rui Malhó ${ }^{1 *}$ \\ 1 Faculdade de Ciências, BiolSI - Biosystems \& Integrative Sciences Institute, Universidade de Lisboa, Lisbon, Portugal, \\ ${ }^{2}$ INIAV - Instituto Nacional de Investigação Agrária e Veterinária, Oeiras, Portugal, ${ }^{3}$ Centro de Estudos Florestais, Instituto \\ Superior de Agronomia, Universidade de Lisboa-Tapada da Ajuda, Lisbon, Portugal, ${ }^{4}$ Instituto Gulbenkian de Ciência, \\ Oeiras, Portugal
}

Allene oxide synthase (AOS) is a key enzyme of the jasmonic acid (JA) signaling pathway. The AOS gene was previously found to be upregulated in an Asian chestnut species resistant to infection by the oomycete Phytophthora cinnamomi (Castanea crenata), while lower expression values were detected in the susceptible European chestnut (Castanea

OPEN ACCESS

Edited by:

Paloma Sanchez-Bel,

University of Jaume I, Spain

Reviewed by:

Álvaro Camisón,

University of Extremadura, Spain

Glória Catarina Pinto,

University of Aveiro, Portugal

*Correspondence:

Susana Serrazina

smserrazina@fc.ul.pt

Rui Malhó

r.malho@fc.ul.pt

Specialty section

This article was submitted to

Plant Pathogen Interactions,

a section of the journal

Frontiers in Plant Science

Received: 12 November 2020

Accepted: 21 January 2021

Published: 15 February 2021

Citation:

Serrazina S, Machado H, Costa RL,

Duque P and Malhó R (2021)

Expression of Castanea crenata Allene

Oxide Synthase in Arabidopsis

Improves the Defense to

Phytophthora cinnamomi.

Front. Plant Sci. 12:628697.

doi: 10.3389/fpls.2021.628697 sativa). Here, we report a genetic and functional characterization of the C. crenata AOS (CCAOS) upon its heterologous gene expression in a susceptible ecotype of Arabidopsis thaliana, which contains a single AOS gene. It was found that Arabidopsis plants expressing CCAOS delay pathogen progression and exhibit more vigorous growth in its presence. They also show upregulation of jasmonic acid and salicylic acid-related genes. As in its native species, heterologous CcAOS localized to plastids, as revealed by confocal imaging of the CcAOS-eGFP fusion protein in transgenic Arabidopsis roots. This observation was confirmed upon transient expression in Nicotiana benthamiana leaf epidermal cells. To further confirm a specific role of CcAOS in the defense mechanism against the pathogen, we performed crosses between transgenic CcAOS plants and an infertile Arabidopsis $A O S$ knockout mutant line. It was found that plants expressing CCAOS exhibit normal growth, remain infertile but are significantly more tolerant to the pathogen than wild type plants. Together, our results indicate that CcAOS is an important player in plant defense responses against oomycete infection and that its expression in susceptible varieties may be a valuable tool to mitigate biotic stress responses.

Keywords: Arabidopsis thaliana, allene oxide synthase, plant defense, gene functional analysis, Phytophthora cinnamomi, Castanea crenata, Castanea sativa

\section{INTRODUCTION}

The European chestnut (Castanea sativa) suffers significant losses in orchard production due to its most dangerous pathogen, Phytophthora cinnamomi. P. cinnamomi is a soil-borne hemibiotrophic oomycete that infects roots in the presence of water through motile zoospores, an infection that can also occur artificially using mycelium inocula (Moralejo et al., 2009; Redondo et al., 2015). From roots, the pathogen progresses through the vascular system, hampering water and nutrient uptake, causing host disease and eventually death (Maurel et al., 2004). 
Among chestnuts, the Asian species exhibit higher resistance to $P$. cinnamomi, particularly the Japanese chestnut, C. crenata. We previously sequenced the root transcriptomes of C. crenata and $C$. sativa upon $P$. cinnamomi inoculation and found differentially expressed genes in $C$. crenata that are candidate defense genes against this oomycete (Serrazina et al., 2015). Among these, allene oxide synthase (AOS) presented a striking expression pattern, being upregulated 5-fold in the resistant species while downregulated 3 -fold in $C$. sativa (Serrazina et al., 2015).

The defense response against soil-borne pathogens in roots begins with microorganism recognition through microbeassociated molecular patterns (MAMP) and/or plant damageassociated molecular patterns (DAMP); both can initiate a plant immune response [reviewed by De Coninck et al. (2014)]. Membrane-bound pattern recognition receptors of the plant recognize M/DAMPs leading to MAMP-triggered immunity (MTI) and, subsequently, cell wall fortification, production of reactive oxygen species (ROS), pathogenesis-related proteins (PR proteins) and secondary metabolites such as phytoalexins (De Coninck et al., 2014). Pathogens that can suppress MTI produce effectors that mask MAMPs, inhibit proteases and thus hamper host responses. Oomycete pathogens can develop haustoria that are able to release effectors into the plant cell. Haustoria are specialized hyphae capable of penetrating the host cell for nutrient uptake from the cytoplasm (Redondo et al., 2015) and correspond to a biotrophic growth of the pathogen (Attard et al., 2010). On the other hand, plants co-evolving with pathogens can develop effector recognition (via R genes) through proteins with a nucleotide-binding (NB) and a leucine-rich repeat (LRR) domain, leading to effector-triggered immunity. This type of immunity is stronger than MTI and can give rise to the hypersensitive response, characterized by programmed cell death. After MTI, root defense responses are regulated depending on the type of threat, with the phytohormones salicylic acid (SA) and jasmonic acid (JA), among others, playing crucial roles in the primary signaling (Chen et al., 2015).

JA and derived metabolites function as signals in the response to several stimuli, including biotic or abiotic stress and wounding, as well as in developmental processes, such as pollen development and anther dehiscence, root growth or fruit ripening (Devoto and Turner, 2005). More recently, Balfagón et al. (2019) described an important role of JA in plant acclimation to intense light and heat stress.

AOS, or Cytochrome P450 74A, is predominantly localized in the plastid membrane (Froehlich et al., 2001) and takes part in the first steps of the JA signaling pathway; lipoxygenase produces 13-hydroperoxy-linoleic acid from membrane lipids, which spontaneously degrades into a keto-hydroxy fatty acid derivative that is transformed into allene oxide by AOS (Chapple, 1998). Allene oxide is rapidly cyclized by allene oxide cyclase to a more stable product, cis-(+)-12-oxo-phytodienoic acid (OPDA) (Laudert and Weiler, 1998). Our previous work pointed to the importance of the JA pathway for the chestnut defense response to $P$. cinnamomi, revealing the differential expression of both JA pathway and JA-induced genes upon C. crenata and C. sativa infection (Serrazina et al., 2015). Camisón et al.
(2019) further reported that jasmonoyl-isoleucine (JA-Ile) levels increase in resistant chestnut roots following infection, while JA-Ile is practically undetectable in non-infected roots.

Despite obvious limitations in applied research, many recent plant-pathogen interaction studies have also taken advantage of Arabidopsis thaliana, due to the plethora of available mutants and its detailed genome annotation. Arabidopsis-Phytophthora pathosystems have been established and used to investigate the role of the JA pathway or related genes in the plant's response to infection, namely Arabidopsis-P. infestans (PajerowskaMukhtar et al., 2008), Arabidopsis-P. parasitica (Attard et al., 2010) and Arabidopsis-P. cinnamomi (Rookes et al., 2008). Studies using the first two systems reported that Arabidopsis mutants impaired in the JA pathway exhibit enhanced pathogen susceptibility, pointing to the involvement of JA in the resistance to Phytophthora. Moreover, Attard et al. (2010) and Rookes et al. (2008) describe different responses of roots and leaves to the pathogen, suggesting that the regulation of defense genes by phytohormones is organ dependent (Chuberre et al., 2018).

In A. thaliana, AOS is encoded by a single gene. Given that our transcriptomic data suggested the involvement of AOS in the resistance of Japanese chestnut to $P$. cinnamomi (Serrazina et al., 2015), we devised a set of functional and molecular studies to test this hypothesis. We resorted to the heterologous constitutive gene expression of $C$. crenata AOS (CcAOS) in A. thaliana plants of the Landsberg erecta (Ler-0) ecotype, known for its susceptibility to P. cinnamomi (Robinson and Cahill, 2003). An Arabidopsis-P cinnamomi pathosystem was developed in which plants were root-inoculated under axenic conditions to analyze the response of transgenic plants expressing CcAOS. To confirm functionality of this heterologous gene expression, we performed subcellular localization analyses of CcAOS-eGFP and genetic crosses of an Arabidopsis aos mutant line with CcAOS.

We show that CcAOS expression in Arabidopsis is able to delay pathogen progression along the root, concomitant with an upregulation of JA- and SA-related genes in transgenic CcAOS plants $24 \mathrm{~h}$ after infection, suggesting that both signaling pathways are involved in the response to $P$. cinnamomi at early stages of host tissue invasion. CcAOS-eGFP was observed in plastids and the expression of CcAOS in aos loss-offunction mutants strengthens a role of the heterologous gene in plant defenses to oomycetes. Together, our results support an important role of C. crenata AOS in biotic stress mechanisms and open new perspectives toward the generation of new C. sativa cultivars.

\section{MATERIALS AND METHODS}

\section{Plant Material and Growth Conditions}

The Arabidopsis thaliana (L.) Heynh. ecotype Landsberg erecta (Ler-0) was used for transformation with the C. crenata AOS gene. For all experiments, Arabidopsis seeds were stratified in water at $4^{\circ} \mathrm{C}$ for $48-72 \mathrm{~h}$ and surface sterilized for $1 \mathrm{~min}$ in $70 \%$ ethanol, $10 \mathrm{~min}$ in $30 \%$ bleach, and $0.5 \%$ Tween 20 , with mechanical mixing, followed by three washes with sterile distilled water. After, seeds were transferred to soil (turf and vermiculite, 3:1 mix) and periodically watered. Arabidopsis plants were grown 
at $22^{\circ} \mathrm{C}$ and $70 \%$ relative humidity, with a $16: 8 \mathrm{~h}$ light: dark photoperiod using walk-in growth chambers (Aralab, Rio de Mouro, Portugal) to promote the reproductive cycle. Growth of Arabidopsis plants for transformation was under a $12: 12 \mathrm{~h}$ light:dark photoperiod to promote vegetative tissue growth and plant robustness for transformation.

Nicotiana benthamiana Domin plants, used for transient transformation of leaf epidermal cells, were cultured from seed in soil (turf and vermiculite, 6:1 mix) and grown at $25^{\circ} \mathrm{C}$ and $70 \%$ relative humidity, with a $16: 8 \mathrm{~h}$ light: dark photoperiod, in a walk-in chamber.

For selection of transformed seeds, stratification was followed by surface sterilization. Seeds were germinated on $9 \mathrm{~cm}$ diameter plates with 0.5X Murashige and Skoog medium (MS medium, Murashige and Skoog, 1962) with 1\% agar, supplemented with 10 $\mathrm{mg} / \mathrm{L}$ of BASTA (Glufosinate-ammonium PESTANAL ${ }^{\circledR}$, Riedelde Haën, Germany). After 7-14 days, all green seedlings were transferred to soil. The mutant genotype for allene oxide synthase (AOS, AT5G42650), acquired from GABI-KAT (GK_624b02, Kleinboelting et al., 2012) was in the Col-0 background and was cultured as above for Arabidopsis transformation, but selection was achieved with $5.0 \mathrm{mg} / \mathrm{L}$ of Sulfadiazine (Sigma-Aldrich, St. Louis, MI, USA). Henceforward the mutant is described as aosGK624b02.

For transgenic plant phenotyping, five seeds were germinated in the upper area of $100 \times 51 \mathrm{~mm}$ squared plates or seedlings were transferred to soil after BASTA selection. For plant inoculation with Phytophthora cinnamomi, assays were performed in vitro and axenically as for plant phenotyping in squared plates.

The GK_624b02 AOS mutant line was crossed to the transgenic CcAOS1 and CcAOS2 lines, which were used as pollen donors to pistils from the mutant. Seeds from F1 and F2 were germinated in the presence of two selection agents, sulfadiazine and BASTA, and plants were transferred to soil and let to auto pollinate. F2 plants were genotyped as described below for the isolation of the transgenic CcAOS lines (primers for AtAOS and T-DNA insertion were as recommended upon https://www. gabi-kat.de/db/primerdesign.php and https://www.gabi-kat.de/ faq/vector-a-primer-info.html respectively). Seeds from F3 were germinated in the same selection conditions and plants grown in vitro for pathogen-inoculation (see below).

Arabidopsis wild type Col-0, aos mutants and aos:CcAOS 2-week-old plants inoculation with $P$. cinnamomi and mycelia progression was performed and analyzed as described below.

\section{Isolation and Cloning of the Castanea crenata Allene Oxide Synthase ORF}

The nucleotide sequence of the $C$. crenata AOS transcript was obtained from the sequenced root transcriptome after P. cinnamomi inoculation (Serrazina et al., 2015). After a BLASTn and comparison of the sequences with highest homology, a prediction of the open reading frame (ORF) and translation to the amino acid sequence was achieved. The CcAOS and Arabidopsis thaliana AOS amino acid sequence were aligned using BioEdit (https://bioedit.software.informer. com, version 7.0.5). The existence and position of the signal plastid peptide was predicted using Localizer (http://localizer. csiro.au/, Sperschneider et al., 2017). Specific primers were designed at the $5^{\prime}$ and $3^{\prime}$ ends of the C. crenata AOS ORF (forward 5'-3' ATGGCATCCACTTCTCTAGCTTTTC, reverse $5^{\prime}-3^{\prime}$ TCAAAAGCTGGCCTTTTTGAG), which was amplified from inoculated C. crenata double stranded cDNA with Phusion High-Fidelity DNA Polymerase (Thermo Fisher Scientific, Waltham, MA, USA), following the manufacturers' instructions. The expected amplification product was run in a $1 \%$ agarose gel, excised and purified with QIAquick Gel Extraction Kit (Qiagen, Hilden, Germany). The product was then cloned in pJET1.2/blunt within CloneJET PCR Cloning Kit (Thermo Fisher Scientific, Waltham, MA, EUA) and sequenced (Stabvida, Caparica, Portugal). After confirming its sequence, the C. crenata AOS ORF was sub-cloned in the pBA-eGFP binary vector, between XhoI and BamHI restriction sites and without the stop codon, resulting in pBA-CcAOS-eGFP. In this vector, $35 \mathrm{~S}$ promoter drives the expression of CcAOS-eGFP and the nucleotide sequence was confirmed by Sanger sequencing.

\section{Plant Transformation}

The pBA-CcAOS-eGFP vector was inserted in the Agrobacterium tumefaciens strain GV3101 and Arabidopsis Ler-0 plants transformed following a modified flower-dip method (drop-by-drop method, Martinez Trujillo et al., 2004). The resulting seeds were germinated in plates supplemented with BASTA for transformant screening. Leaf samples from putatively transformed 1-month-old plants were genotyped to verify the presence of C. crenata AOS ORF and eGFP in the genomic DNA, with KAPA3G Plant PCR Kit (Kapa Biosystems, Wilmington, MA, USA), following Section 2: Direct PCR. The primers used to amplify eGFP were: forward $5^{\prime}-3^{\prime}$ GGGACGTCATGGTGAGCAAGG and reverse $5^{\prime}-3^{\prime}$ CGTCCATGCCGAGAGTGATCC. Transformed plants were selected and let self-pollinate to generate F1. Plants of the F1 and F2 generation were also screened with BASTA and genotyped to confirm a stable transformation in each independent transformed line. Seeds, seedlings and plants derived from the F2 were used for subsequent assays (heterologous protein localization, phenotyping and inoculation assays with P. cinnamomi).

For transient expression of the fusion CcAOS-eGFP protein in Nicotiana benthamiana, 5-6-week-old plants were used and leaf infiltration with Agrobacterium strain GV3101 (Sparkes et al., 2006) harboring pBA-CcAOS-eGFP or the empty pBA-eGFP vector performed.

\section{Subcellular Localization of the Castanea crenata AOS Protein in Arabidopsis}

Roots of 1-week-old Arabidopsis lines transformed with CcAOSeGFP or with the corresponding empty vector were observed in a Leica SP-E confocal microscope (Leica Microsistemas, Carnaxide, Portugal) with the settings described below and the $488 \mathrm{~nm}$ laser line.

In transiently transformed $N$. benthamiana, sections of leaves from the infiltrated areas were observed 2, 3, and 4 days after infiltration. Imaging was achieved in a Leica SP8 confocal 
microscope (Leica Microsistemas, Carnaxide, Portugal). Optical sections (ca $2 \mu \mathrm{m}$ thick) were acquired using a x63 ACS APO water objective $(\mathrm{NA}=1.15),<10 \%$ laser intensity (488 and $552 \mathrm{~nm}$ laser lines) and operating in the mode $1,072 \times 1,072$, $600 \mathrm{~Hz}$ (c. $0.3 / \mathrm{s}$ per frame). Image or Z-stack acquisition was linear, ensuring no signal bleed through.

Images were processed using the Image J software (https:// imagej.nih.gov/ij/). All observations were repeated twice with at least three individual plants per genotype.

\section{Phenotypical Analysis of Transgenic Arabidopsis Lines}

Plants were grown as described above, with growth and root parameters (rosette area; dry weight; primary root length; lateral root number; root hair number; root hair length) being measured on images of 1-month (potted plants) and 13-day old plants (in vitro plants), respectively, using the Image J software. Fifteen plants per genotype were considered in three independent assays.

Flowering time, silique length and silique number were scored in 15 plants per genotype, with three flowers/siliques being collected per plant in three independent assays.

Results represent means \pm standard deviation, and Student's $t$-test was used for statistical analysis.

\section{Analysis of Castanea crenata AOS Expression Levels in Transgenic Arabidopsis Lines}

Total RNA was isolated from plants of wild type or CcAOS transformed lines using the RNeasy Plant Mini Kit (Qiagen, Hilden, Germany), followed by treatment with Turbo DNase kit (Thermo Fisher Scientific, Waltham, MA, USA). Three biological replicates were prepared, each one with six plants per condition. Total RNA $(3.6 \mu \mathrm{g})$ was used as template for reverse transcription with RevertAid H Minus Reverse Transcriptase (Thermo Fisher Scientific, Waltham, MA, USA) and primed with an oligo(dT) primer. Specific primers for CcAOS were designed with PrimerSelect 5.03 (DNASTAR Inc., Madison, WI, USA) (forward 5'-3' CACGCGTCGATTTATTGTCC and reverse 5'3' TTTGGTGGGTTCGGCTTGTT).

Each cDNA was diluted 1:40 and $4 \mu \mathrm{L}$ (18 ng) used per reaction, in a $25 \mu \mathrm{L}$ final volume using Maxima SYBR Green qPCR Master Mix kit (Thermo Fisher Scientific, Waltham, MA, USA). A final concentration of $0.2 \mu \mathrm{M}$ of each primer was used in a StepOne Real-Time PCR system (Applied Biosystems, Foster City, California, USA). Quantitative PCR (qPCR) reactions started with a denaturation step at $95^{\circ} \mathrm{C}$ for $10 \mathrm{~min}$ followed by 40 cycles of denaturation at $95^{\circ} \mathrm{C}$ for $15 \mathrm{~s}$ and annealing temperature $\left(60^{\circ} \mathrm{C}\right)$ for $30 \mathrm{~s}$. Each set of reactions included a no template control and two technical replicates. Dissociation curves were used to analyze non-specific PCR products.

To normalize expression data, ACTIN2 was used. Oligos were forward $5^{\prime}-3^{\prime}$ GGTATTGTGCTGGATTCTGG and reverse $5^{\prime}-3^{\prime}$ CGCTCTGCTGTTGTGGTGA. Annealing temperature was $60^{\circ} \mathrm{C}$. 18srRNA was also used for normalization with identical results to ACTIN2. Oligos were forward 5'-3' AGTCGGGGGCATTCGTATTT and reverse 5'-3' ATCCCTGGTCGGCATCGTTT.

Gene expression was calculated using the $\Delta \Delta \mathrm{CT}$ method (Schmittgen and Livak, 2008). The highest level of expression in transformed line CcAOS2 was used as calibrator (set to 1) to which all the other samples were compared. Student's $t$-test was used for statistical analysis.

\section{Analysis of Castanea crenata AOS Protein Levels in Transgenic Arabidopsis Lines}

Two-week-old plants transformed with CcAOS or the empty vector were first checked for the presence of AOS-eGFP or eGFP expression with a Olympus BX51 fluorescence microscope (Labocontrole, Lisbon, Portugal) equipped with a 470-490/DM505/LP515 filter, and a TIS 2MP DFK23U274 RGB camera (Infaimon, Aveiro, Lisbon, Portugal). A maximum of $100 \mathrm{mg}$ of plants from each genotype was stored in triplicate at $-80^{\circ} \mathrm{C}$. Tissue was grinded with liquid nitrogen before the addition of $300 \mu \mathrm{L}$ of lysis buffer. RIPA buffer (radioimmunoprecipitation assay buffer; https://www.abcam. com/protocols/sample-preparation-for-western-blot) was used to obtain a protein extract with plastid proteins. Protease Inhibitor Cocktail (Sigma-Aldrich, St. Louis, MI, USA) was added freshly to the lysis buffer as recommended.

The total protein in each extract was quantified using the Bio-Rad Protein Assay (Bio-Rad, Hercules, CA, USA), and $24 \mu \mathrm{g}$ run in a $10 \%$ SDS-PAGE. A monoclonal antibody for eGFP (Roche, Basel, Switzerland) was used for Western blotting analysis at 1:10,000 dilution, followed by Peroxidase Affinipure Goat Anti-Mouse IgG (Jackson Immunoresearch, Ely, UK) at 1:10,000, before detection with NZY ECL Supreme HRP substrate (NZYtech, Lisboa, Portugal).

\section{P. cinnamomi Inoculation Assays}

Liquid cultures of $P$. cinnamomi (isolate IMI 340340 from the University of Trás-os-Montes and Alto Douro) were prepared in $50 \mathrm{~mL}$ of $1 \% \mathrm{PDB}$ before incubation in a rotary shaker at $24^{\circ} \mathrm{C}$ and $100 \mathrm{rpm}$ for $48 \mathrm{~h}$. Mycelia were then resuspended in $5 \mathrm{~mL}$ of $1 \% \mathrm{PDB}$ and blended at high speed for $1 \mathrm{~min}$. The concentration of mycelial fragments in the stock suspension was quantified with the aid of a hemocytometer and the mycelial suspension adjusted with $1 \%$ PDB to reach final concentration of $\sim 1.0 \times$ $10^{4}$ fragments/mL.

Two-week-old wild-type Ler-0 and transgenic CcAOS lines, wild-type Col-0, aos mutants and F3 aos:CcAOS Arabidopsis plants, growing axenically in vitro (in 0.5X MS medium), were inoculated at the root cap with $10 \mu \mathrm{L}$ of mycelia fragment suspension. In mock inoculations, potato dextrose broth $1 \%$ was added. Plates were covered with a black cloth overnight to promote infection. The following day, the cloth was removed, the plates were shaded in the root area and placed vertically.

Photographs were taken at 3, 6, and 9 days after inoculation and measurements of mycelia progression along root performed using the Image J software (https://imagej.nih.gov/ij/). Mycelia progression was also observed microscopically at $1,2,3,4,5$, and 6 days after inoculation; oomycete tissues in Arabidopsis roots were dyed with trypan blue $0,05 \%$ in a solution of lactoglycerol 
[lactic acid, glycerol, and water (1:1:1)] for $5 \mathrm{~min}$ and rinsed in lactoglycerol before observation in an Olympus BX51 microscope coupled with a TIS 2MP DFK23U274 RGB camera.

At least 10 plants per genotype were analyzed in three independent inoculation assays.

\section{Quantification of in Planta P. cinnamomi Growth}

Genomic DNA was isolated from all Arabidopsis genotypes (wild-type Ler-0 and the 3 transgenic lines) at 6 and 9 days after inoculation using the DNeasy Plant Mini Kit (Qiagen, Hilden, Germany). Three biological replicates were prepared, each one with five plants per genotype and time-point.

Quantification of $P$. cinnamomi growth in planta was achieved through the quantification of the Pyruvate, phosphate dikinase gene (Pdk, GenBank assession FJ493007.1) by qPCR, based on Eshragui et al. (2011a). Primers for Pdk were forward 5'-3' GACGAGAGCGAGACAAGAA and reverse $5^{\prime}-3^{\prime}$ CAAACGCACAAACGCACAC, and the melting temperature was $58^{\circ} \mathrm{C}$. The amount of genomic DNA used per reaction was $1.84 \mathrm{ng}$ and the reaction mix is described above. Each set of reactions included a no template control and two technical replicates. Monensin sensitivity 1 (Mon1, At2g28390.1) of the SAND family protein served as a reference gene (Schlaeppi et al., 2010), using primers forward 5'-3' GTGGCGGCGATGATAATGAT and reverse 5' $-3^{\prime}$ CTAGTTCCCGCCACACCTT. RNA Processing Factor 3 (RPF3 Atlg62930.1) was also used for normalization (Czechowski et al., 2005) yielding identical results to Mon1. Oligos were forward 5'-3' GAGTTGCGGGTTTGTTGGAG and reverse 5'-3' CAAGACAGCATTTCCAGATAGCAT.

$P$. cinnamomi biomass in inoculated plants was calibrated with the level of inoculated wild type Ler-0 plants. The experiment was repeated in three independent assays, and Student's $t$-test was used for statistical analysis.

\section{Expression Analysis of Genes Related to the Jasmonic and Salicylic Acid Pathways}

Total RNA was isolated from root and aerial tissue of wild type Ler- 0 and the three transgenic CCAOS lines at 3, 12, and $24 \mathrm{~h}$ after inoculation, using the RNeasy Plant Mini Kit (Qiagen, Hilden, Germany), followed by treatment with Turbo DNase kit (Thermo Fisher Scientific, Waltham, MA, USA). Three biological replicates were prepared, each one with five plants per genotype, time-point and tissue type. Total RNA, cDNA, reaction reagents and cycling were as described above, and 5.9 ng of cDNA were used per reaction. Each set of reactions included a no template control and two technical replicates. Primer sequences for each gene are listed in Supplementary Table 2.

To normalize expression data, Mon1 and RPF3 were used, and gene expression levels were calculated as described above and calibrated with the respective mock-inoculated sample at each given time-point after inoculation. Results are from three independent assays, and Student's $t$-test was used for statistical analysis.

\section{RESULTS}

\section{Transgenic Arabidopsis Plants Expressing CcAOS Exhibit Slightly Accelerated Development}

Arabidopsis wild type plants from the ecotype Landsberg erecta (Ler-0), which are highly susceptible to the oomycete $P$. cinnamomi (Robinson and Cahill, 2003), were transformed with the AOS gene from Castanea crenata (CcAOS). According to our sequencing and in silico analysis, the CcAOS ORF has $1,581 \mathrm{nt}$ and is predicted to encode 527 amino acids, with 58.7 $\mathrm{kDa}$ molecular weight and $9.01 \mathrm{pI}$. After a BLASTp in NCBI, the most related sequences were found to be from Quercus suber, Morus notabilis and Camellia sinensis, showing 96, 80, and $76 \%$ identity, respectively (data not shown). The CcAOS amino acid sequence shares $68 \%$ identity with the A. thaliana AOS (At5g42650.1) (Supplementary Figure 1). The CcAOS ORF was cloned upstream of the eGFP sequence under the control of the constitutive CaMV35S promoter (Figure 1A).

Three independent Arabidopsis lines stably transformed with pBA-CcAOS-eGFP were isolated (Figures 1B,C). Subsequent generations of these plants were analyzed and compared to the wild type to assess the effects of heterologous CcAOS expression. Transgenic CCAOS Arabidopsis plants were morphologically very similar to the wild type although with taller flower stalks (Figure 2A) and larger rosettes (Supplementary Table 1). Likewise, CcAOS flowers displayed no visible defects (Figure 2B), but developed earlier (Supplementary Table 1) and, upon selffertilization, generated slightly smaller siliques with fewer seeds (Figures 2C,D). The root system of transgenic CcAOS plants also appeared normal (Figure 2E) though exhibiting longer primary root length and more lateral roots $(P<0.05)$ (Supplementary Table 1).

\section{CcAOS Contains a Signal Peptide and Localizes to the Plastid}

AOS was reported to be a plastid protein in plants (Tijet and Brash, 2002). We used an in silico tool to predict transit peptides in the CcAOS sequence, and a plastid signal peptide was found encoded in amino acids 1-21 (Supplementary Figure 1). Three independent transgenic CcAOS Arabidopsis lines were used for subcellular localization analysis of the CcAOS-eGFP fusion protein in roots of 1-week-old seedlings. Plants transformed with the pBA-eGFP binary vector alone were used as a control and compared to the three CcAOS lines. Observations were similar in all lines, but to reduce the possibility of artifactual localization resulting from overexpression, we focused on data stemming from line 3, which exhibited lower CcAOS levels (Figure 1C).

As reported previously and predicted by the presence of the signal peptide, CcAOS-eGFP localization was consistent with accumulation in plastids (Figures $\mathbf{3 A}, \mathbf{B}$ ) - sparse punctuated fluorescence of $\sim 1-3 \mu \mathrm{m}$ near the membrane of highly vacuolized cells, which were not observed in control experiments (Figures 3C,D). This pattern is similar to observations made in Physcomitrella patens (Scholz et al., 2012), Vitis vinifera (Dumin et al., 2018), and Camelia sinensis (Peng et al., 2018). 
A

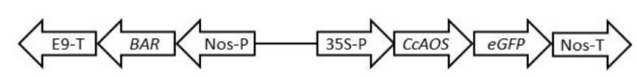

B

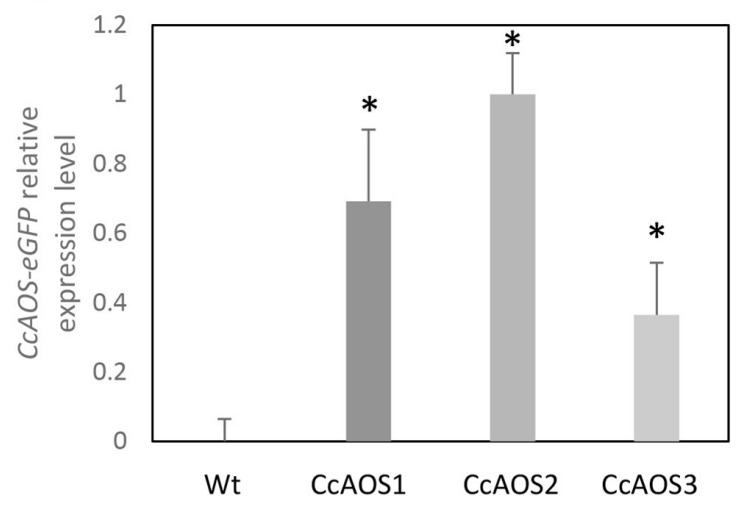

C

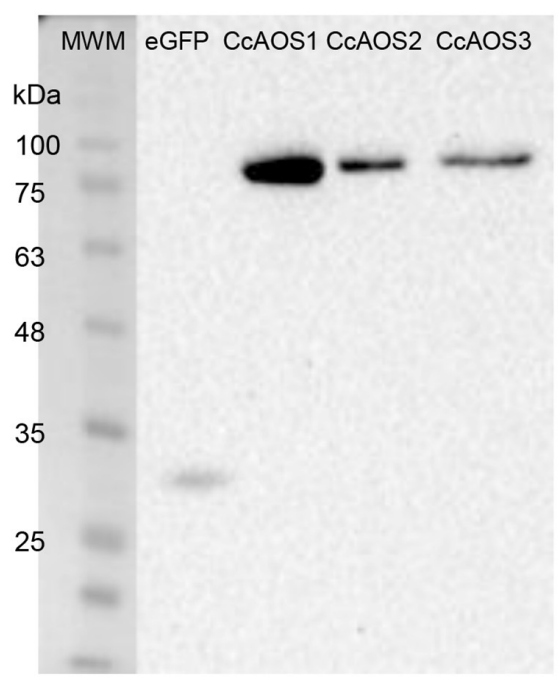

FIGURE 1 | Molecular characterization of transgenic Arabidopsis plants expressing CCAOS. (A) Schematic diagram of the CCAOS-eGFP construct used for transformation. 35S-P: CaMV 35S RNA promoter; Nos-T: Nopaline synthase terminator; Nos-P: Nopaline synthase promoter; BAR:

Phosphinothricin N-acetyltransferase coding sequence; E9-T: pea rbcS-E9 terminator. (B) Relative expression levels of CCAOS-eGFP in 2-week-old transgenic plants. Wild type Ler-0 was used as negative control. Expression levels were normalized to the Actin2 (At3g18780) reference gene. The highest level of expression in CCAOS2 was set to 1 and used as calibrator. Error bars represent the standard deviation of the mean $(n=3)$. Asterisks indicate significant differences in the expression between transformed plants with $P<$ 0.005 ( $t$-test). (C) CcAOS-eGFP protein expression in 2-week-old transgenic plants. An eGFP monoclonal antibody was used in hybridization and plants transformed with the empty vector were used as positive control for eGFP (2nd lane). Molecular weights: eGFP 27 KDa; CcAOS-eGFP 77 KDa. Image representative of three independent experiments.

The expression of CcAOS-eGFP in roots was significantly lower than in the aerial tissues. As imaging of the Arabidopsis small but dense leaves is recognizably difficult, we also used

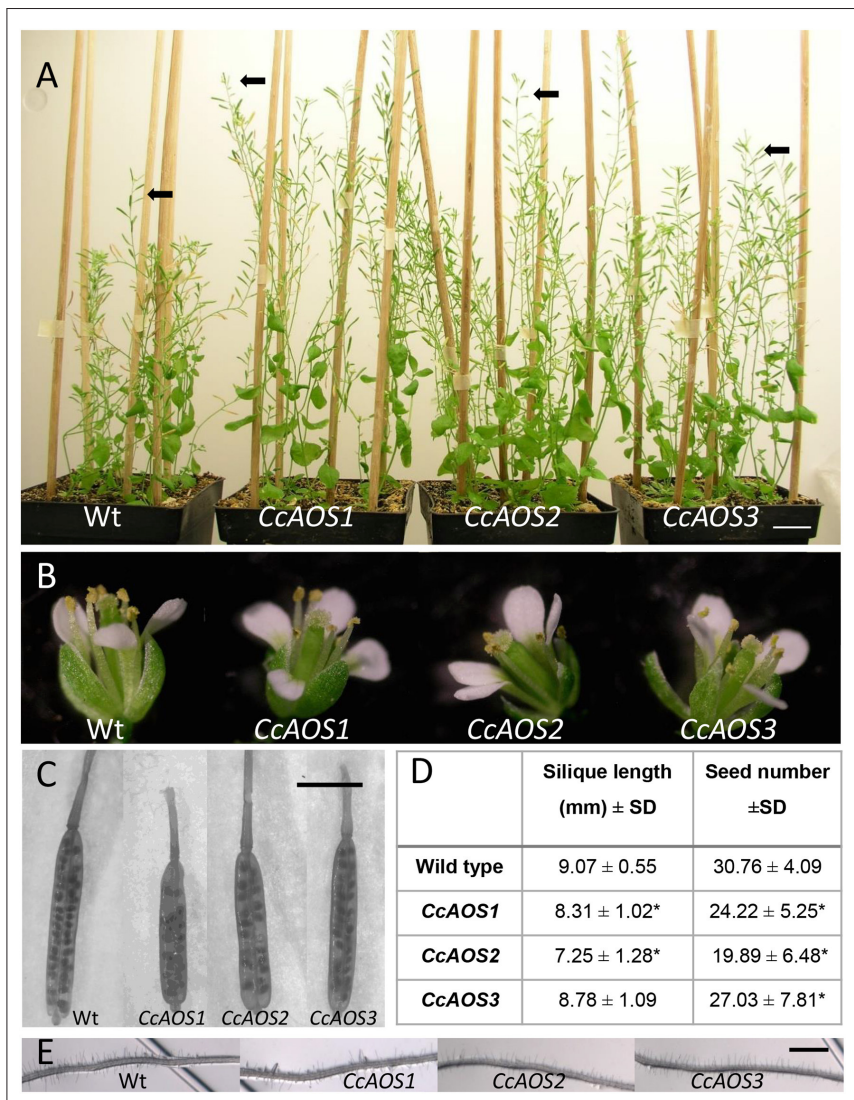

FIGURE 2 | Phenotypical characterization of transgenic Arabidopsis plants expressing CCAOS-eGFP. (A) Two-month-old wild type (Ler-0) and transgenic CCAOS plants. Arrowheads indicate flower stalks' height. Scale bar $=2 \mathrm{~cm}$.

(B) Detail of flowers from plants depicted in (A). (C) Detail of siliques collected from plants depicted in (A). Bar $=5 \mathrm{~mm}$. (D) Average silique length and seed number ( $\pm \mathrm{SD} ; n=3$ ) collected from plant lines depicted in (A). Asterisks indicate significant differences when compared to Ler-0 plants with $P<0.05$ (t-test). (E) Detail of roots from plants depicted in A. Scale Bar $=100 \mu \mathrm{m}$.

transiently transformed Nicotiana benthamiana epidermal leaf tissue for a detailed analysis of the subcellular localization and dynamics of CcAOS-eGFP fluorescence. The same pBACcAOS-eGFP plasmid used to transform Arabidopsis plants was infiltrated into $N$. benthamiana leaves and observations performed after 4 days (Figures 4A-F). CcAOS-eGFP was found to also concentrate in punctuated structures that co-localize with chloroplasts (Figures 4A-C) and decorate their outer regions. Interestingly, alongside with bright fluorescent spots of CcAOSeGFP accumulation, we could also register a faint reticulatelike distribution (Figure 4C arrows, Supplementary Figure 2) that was not present in control experiments. This suggests that the trafficking of CcAOS to the plastids may involve and/or be mediated by the endomembrane compartment.

\section{Expression of CcAOS in Arabidopsis Reduces Susceptibility to $P$. cinnamomi Infection and Delays Pathogen Progression}

To assess if the constitutive expression of CcAOS affects pathogen progression along the root, we developed an axenic assay where 

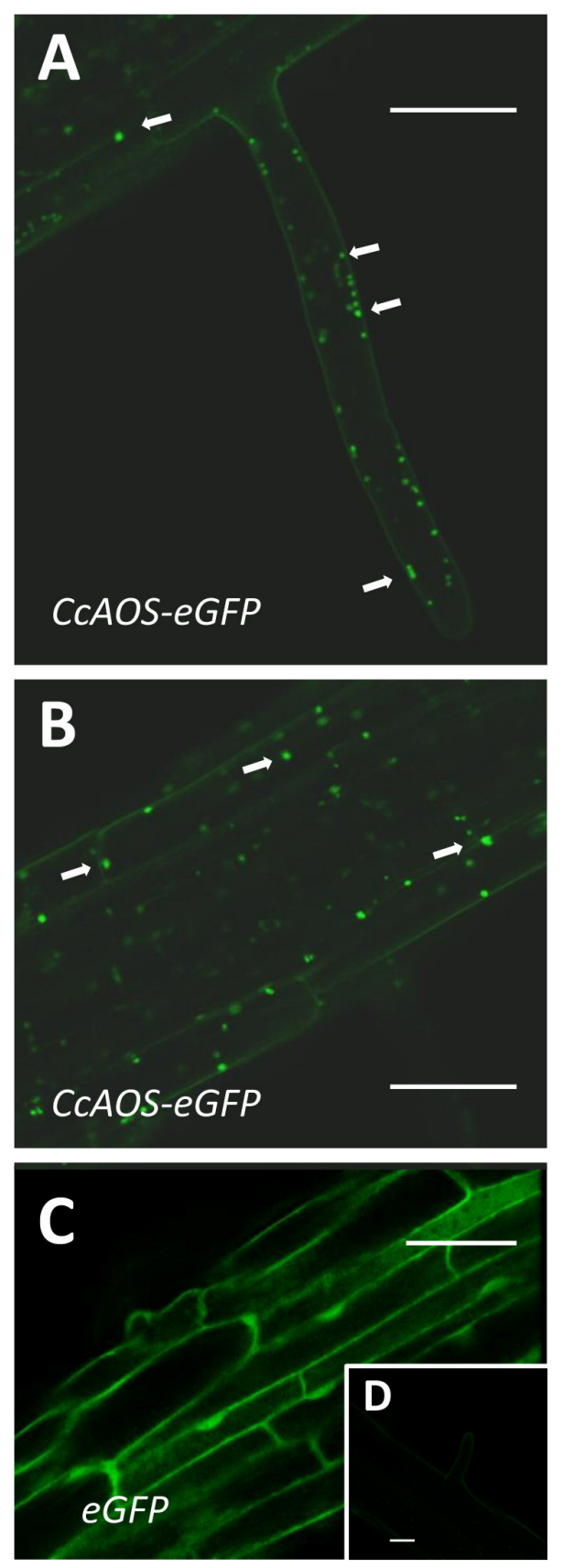

FIGURE 3 | Sub-cellular localization of stable CcAOS-eGFP expression in roots of $A$. thaliana. Images (from line CCAOS3) are representative of the three transgenic lines. Scale Bars $=30 \mu \mathrm{m}$. (A,B) CcAOS-eGFP expression in root hairs (A) and cortical tissue (B) of the primary root. Fluorescence accumulates in plastids (arrows). (C) Control expression of eGFP. (D) Non-transformed cortical tissue.

2-week-old Arabidopsis plants growing in 0.5X MS media were inoculated at the root cap with mycelia fragments of a $P$. cinnamomi virulent strain (Figure 5). Macroscopically, growth of mycelia along the root system was observed from 3 days after inoculation (d.a.i.) and their progression scored at 3, 6, and 9 d.a.i., after which the pathogen was able to colonize the root completely. The plants transformed with CcAOS showed the lowest percentage of root colonization reaching a maximum of $58 \%$ at 9 d.a.i., whereas wild type plants exhibited similar pathogen progression values at 6 d.a.i. (Figure 5A). After colonization of the entire root, mycelia accumulated in the aerial part of the plant, with their density being notably lower in transgenic CcAOS plants (Figure 5B). No necrosis was observed in roots, with leaf chlorosis being notable as soon as mycelia reached the base of the stem. No plant survival was detected 2 months after inoculation. In addition to physical progression along the root, we also evaluated pathogen load via quantification of a $P$. cinnamomi gene (Pyruvate, phosphate dikinase, Pdk) at days 6 and 9 after inoculation. As expected, the amount of pathogen DNA was significantly higher for wild type plants at both time points (Figure 5C).

The progression of $P$. cinnamomi mycelia along roots was also cytologically followed during the 1st week after inoculation. Until 1 day after inoculation, finger-like hyphae on epidermal cells, identified as haustoria, were observed in non-transformed and $C c A O S$ transformed roots (Figures 6A,B). Two days after inoculation, hyphae reached the cortex both intercellularly and intracellularly (Figures 6C,D), and we identified hyphal aggregations corresponding to stromata. These structures can store nutrients obtained from the host, resulting in de novo production of mycelium (and spores) when conditions are favorable (Willetts, 1997). At this time point, unlike with CcAOS plants, hyphae were frequently observed deep in the wild type root, associated with xylem vessels in vascular tissue (Figure 6C). None of the described pathogen structures were observed in association with tissue necrosis.

These results show that the AOS gene from C. crenata delays $P$. cinnamomi progression in planta, when constitutively expressed in Arabidopsis. However, a direct correlation between quantity of $C c A O S$ transcript or protein expressed and the effect on pathogen infection was not observed in our three plant lines. This could represent either biological or technical variability, but it is also possible that the plant's net response reflects an effect of CcAOS over-expression on other metabolic parameters.

\section{Genes Related to Jasmonic Acid and Salicylic Acid Pathways Are Upregulated in Transgenic CcAOS Arabidopsis Plants}

The data collected supports a relevant role for CcAOS against $P$. cinnamomi resistance. To gain insight into the underlying defense mechanisms, we analyzed the expression of genes related to the JA and SA pathways, given their recognized involvement in plant responses to biotic stresses (Clarke et al., 2000; Betsuyaku et al., 2018). The early defense responses of Arabidopsis against Phytophthora pathogens are known to differ between root and leaves (Robinson and Cahill, 2003; Rookes et al., 2008; Attard et al., 2010). We therefore evaluated differential gene regulation in root and leaf tissues. The selection of the time points $-3,12$, and $24 \mathrm{~h}$ after inoculation (h.a.i.) - was based on the observation of haustoria as early as 1 day after inoculation. Haustoria are structures of cell invasion that correspond to pathogen initial biotrophic growth and, according to Attard et al. (2010), SA- and 

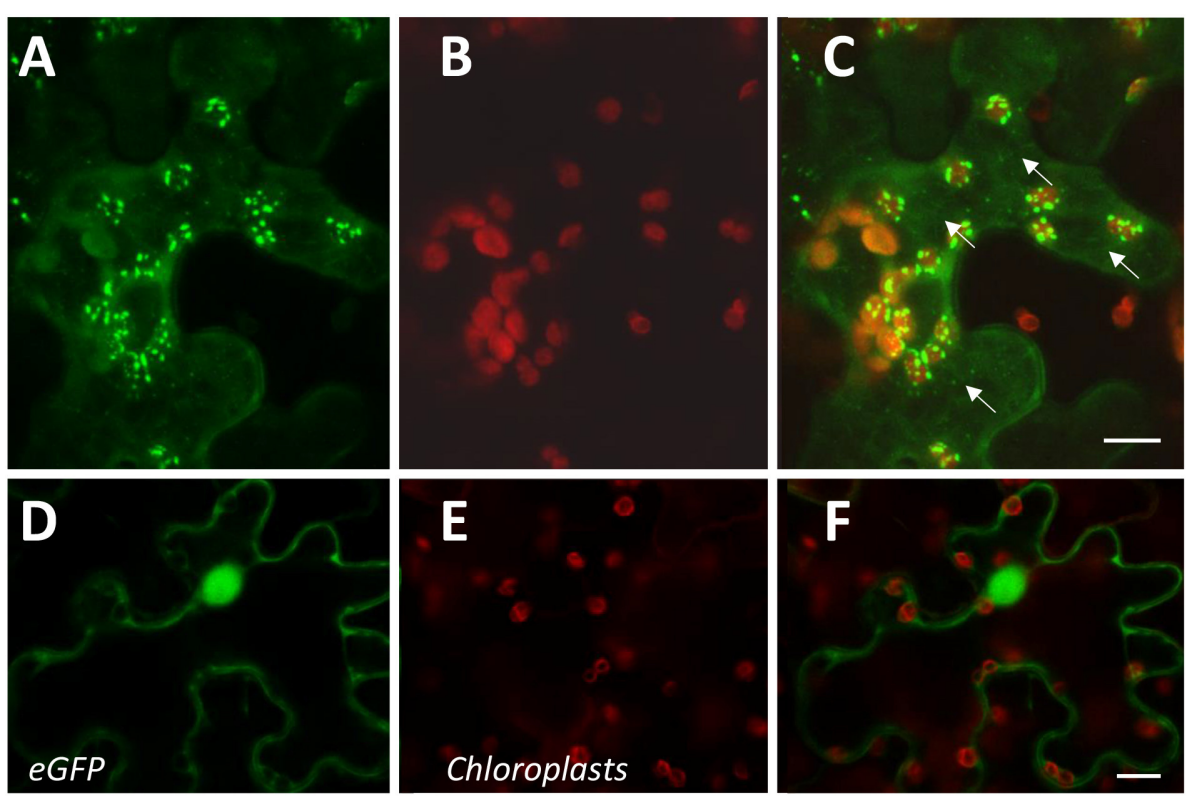

FIGURE 4 | Sub-cellular localization of transient CcAOS-eGFP expression in leaves of $N$. benthamiana. Scale Bars = $10 \mu \mathrm{m}$. (A-C) CcAOS-eGFP (A), chlorophyll autofluorescence (B), and merged image (C). (D-F) Control with eGFP (D), chlorophyll autofluorescence (E), and merged image (F).

JA-signaling pathways are promptly triggered when the oomycete penetrates roots.

The expression of the endogenous AtAOS gene was first assessed to verify transcript fluctuations in the transgenic plant lines. Figure 7 shows a similar transcript profile in wild type and transgenic $C c A O S$ plants, with a significant upregulation in leaves at 24 h.a.i. but not in roots. The similar AtAOS expression pattern in the wild type and in plants constitutively expressing CcAOS, suggests a non-deleterious effect of the heterologous protein in the morphology and development of transformed plant lines.

Previously, we reported significant differential expression of two JA-marker genes, LOX1 and JAZ10, in Castanea upon P. cinnamomi inoculation (Serrazina et al., 2015). Lipoxygenase1 (LOX1) is a 9S-lipoxygenase that precedes AOS in the JA pathway and was found to play an important role in plant defense against pathogens (Vellosillo et al., 2007). Here we found that LOX1 shows a significant upregulation only 24 h.a.i. in leaves, particularly in wild type plants (Figure 7). By contrast, JAZ10 exhibited a significant upregulation 24 h.a.i. in leaves of CcAOS plants, while in the wild type this increase was incipient. JAZ10 is a member of the JASMONATE-ZIM-DOMAIN family which reportedly negatively regulates the JA defense response, promoting growth (Guo et al., 2018). In the susceptible C. sativa, the JAZ10 transcript was found to be downregulated upon P. cinnamomi inoculation (Serrazina et al., 2015).

A downstream marker of JA pathway, PLANT DEFENSIN 1.2 (PDF1.2) was previously tested in the interaction Arabidopsis - P. cinnamomi (Rookes et al., 2008). Here we observed a significant upregulation of $P D F 1.2$ after P. cinnamomi inoculation in leaves of $C c A O S$ plants at a very early time-point (3 h.a.i.), which was only moderately detected in wild type susceptible leaves. This further confirms the involvement of CcAOS and the JA pathway in the defense responses. Together, our analysis of JA-related genes suggests that the over-expression of CcAOS stimulates the JA pathway (downstream of AtAOS), activating plant defenses while alleviating growth reduction, thus allowing the plant to reach the reproduction phase.

Our previous work had also suggested that the SA pathway plays an important role in the local and systemic defense responses to $P$. cinnamomi (Serrazina et al., 2015). We thus included two SA-related genes in the analysis of transgenic CcAOS plants: NONEXPRESSER OF PR GENES 1 (NPR1) and PATHOGENESIS-RELATED GENE 5 (PR5). NPR1 is referred to as a regulator of the interaction between the $\mathrm{SA}$ and JA pathways (Proietti et al., 2018) and has been used as a SA-marker gene in the Arabidopsis- $P$. cinnamomi interplay (Rookes et al., 2008). Here we found minor differences in NPR1 expression between mock and pathogen-inoculated plants ( $<2$-fold up- or down-regulation, Figure 7), suggesting that at the tested time-points regulation of JA signaling by SA is not achieved at the NPR1 level. On the other hand, PR5 encodes a thaumatin-like protein and was used by Eshragui et al. (2011b) as a defense SA-marker gene in P. cinnamomiinfected Arabidopsis. Our data show that the expression of PR5 in wild type threatened plants is downregulated in roots, exhibiting upregulation only $24 \mathrm{~h}$ after inoculation of leaf tissues. By contrast, inoculated transgenic CcAOS plants exhibited PR5 upregulation $3 \mathrm{~h}$ after inoculation both in roots and leaf tissues. These results suggest that the constitutive expression of CcAOS in Arabidopsis promotes the expression of pathogenesis-related proteins through the crosstalk between JA and SA pathways in a NPR1-independent manner. 

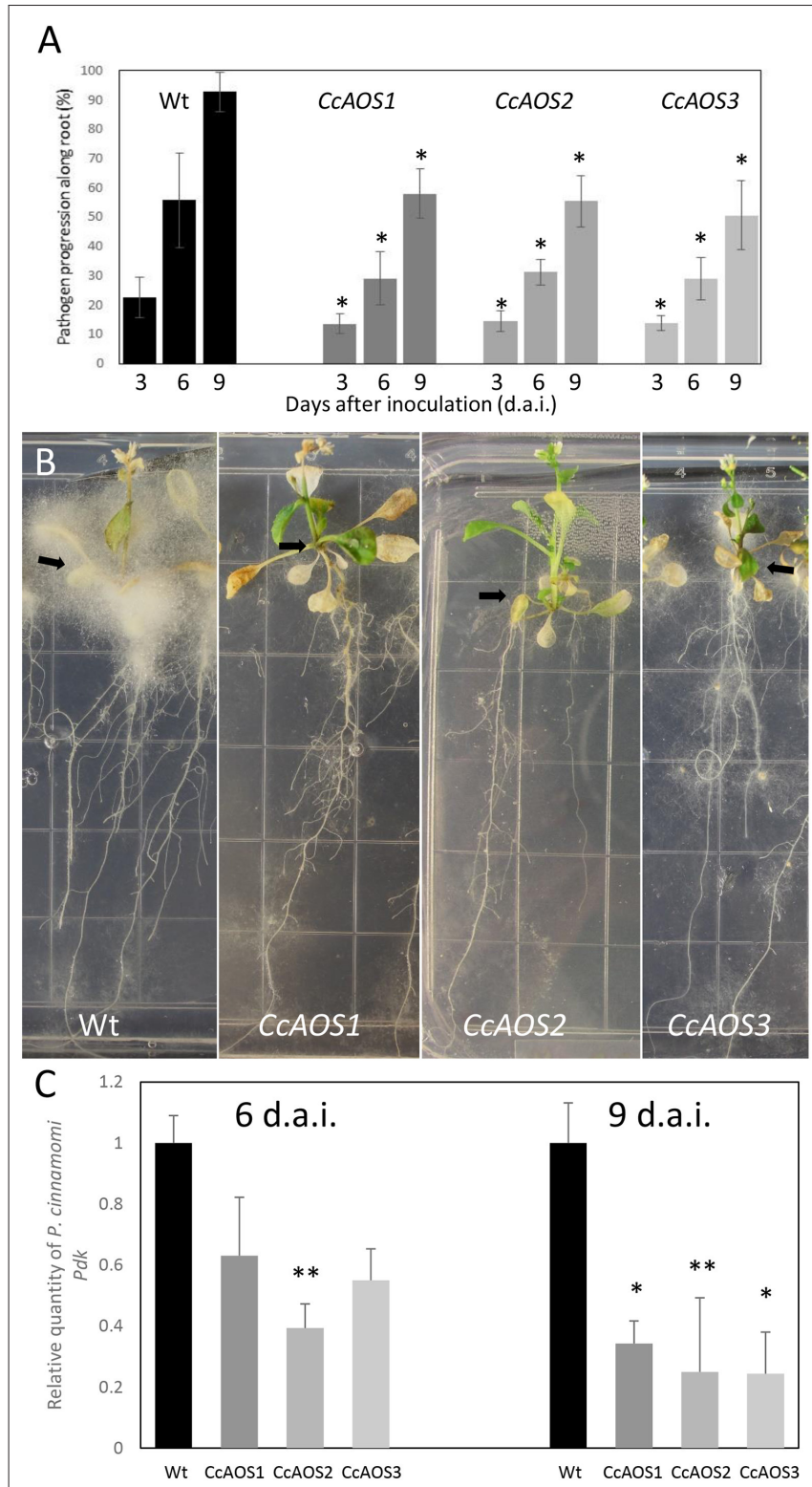

FIGURE 5 | Phytophthora cinnamomi progression in transgenic Arabidopsis plants expressing CCAOS. (A) Percentage of $P$. cinnamomi progression along roots of inoculated plants 3,6 , and 9 days after inoculation. Asterisks refer to significant differences from wild type Ler-0 at each time point $(P<0.001$, $t$-test). Error bars represent the standard deviation of the mean $(n=3)$. (B) Mycelia accumulation around the plant's aerial part (arrows), 3 weeks after inoculation. Grid squares: $1.4 \mathrm{~cm} /$ side. (C) Relative amount of the pathogen gene $P d k, 6$ and 9 days after inoculation (d.a.i.). Values were calculated relative to Ler-0 wild type inoculated plants and normalized to the reference genes Mon1 and RPF3. Asterisks refer to significant differences from the wild type at each time-point, with $P<0.005\left(^{*}\right)$ and $P<0.05\left(^{* *}\right)$ (t-test). Error bars represent the standard error of the mean $(n=3)$.

Noteworthily, despite CcAOS plants showing reduced susceptibility to $P$. cinnamomi, the susceptible nature of both the transgenic and wild type genotypes is reflected in the general downregulation of all analyzed genes at 12 h.a.i., which may

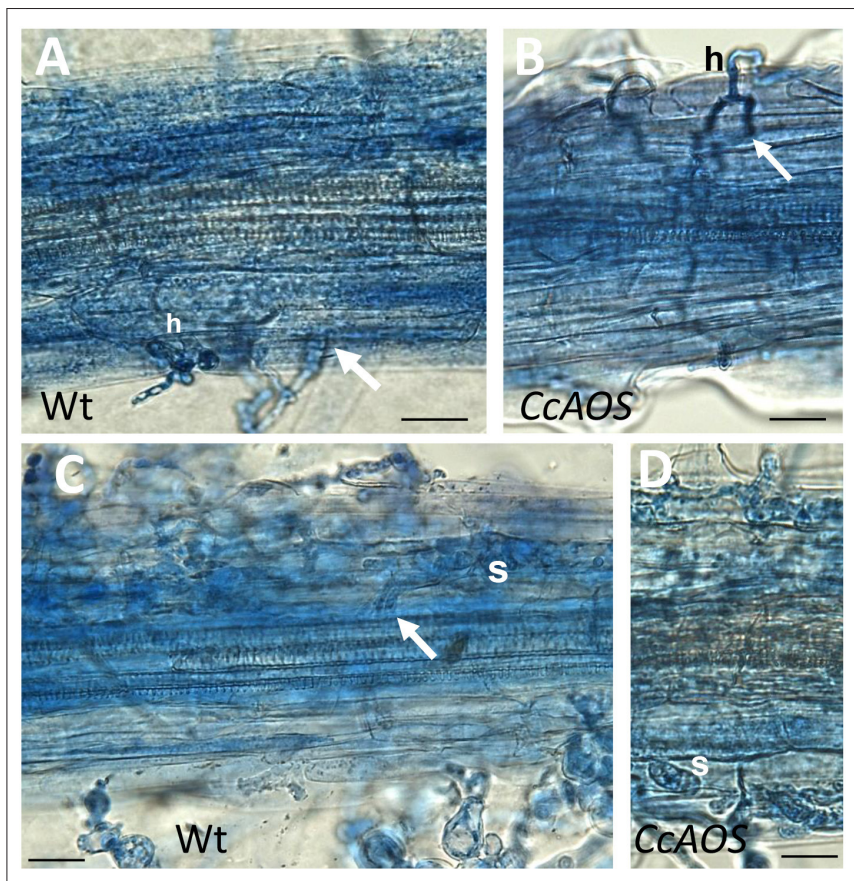

FIGURE 6 | Cytological analysis of transgenic CCAOS Arabidopsis roots inoculated with $P$. cinnamomi. Images (from line CCAOS1) are representative of the three transgenic lines. Scale Bars $=30 \mu \mathrm{m}$. (A,B) One day after inoculation, mycelia develop haustoria (h) on epidermal cells of Ler-0 wild type (A) and transgenic CCAOS (B) roots. Arrows indicate hyphae penetrating the cortex intercellularly. (C,D) Three days after inoculation, stromata (s) develop in cortical cells and hyphae can be observed in the stele (arrow).

correspond to an important stage of pathogen hijacking of JA and SA signaling through its effectors (Herlihy et al., 2019).

\section{The Arabidopsis and Chestnut AOS Fulfill Distinct Roles in planta}

In Arabidopsis, loss of function of the single AOS gene (AT5G42650) was previously reported to cause male sterility (Park et al., 2002; von Malek et al., 2002). Here we retrieved an Arabidopsis AOS mutant allele, aosGK624b02, from the Gabi-Kat collection of T-DNA insertion mutants. Such mutant, with Col-0 background, has low susceptibility to P. cinnamomi (Robinson and Cahill, 2003). The phenotype reported for this aos mutant (Park et al., 2002; von Malek et al., 2002), was confirmed in our aosGK624b02 plants which developed anthers with small filaments, incipient siliques and exhibited disturbed pollen maturation and viability (Supplementary Figure 3). To investigate a potential role of the CcAOS protein in plant fertility, F1 plants obtained from the genetic crossing of the aos GK624b02 mutant (pistils) and a CcAOS transgenic line (pollen) were let to self-pollinate and produced fertile seeds. Upon selection with two selection agents, sulfadiazine and BASTA, 28 resistant plants from F2 were obtained and grown in soil to maturity. From those, 22 displayed regular anthers that were able to generate viable pollen and to pollinate the stigma, resulting in normal 


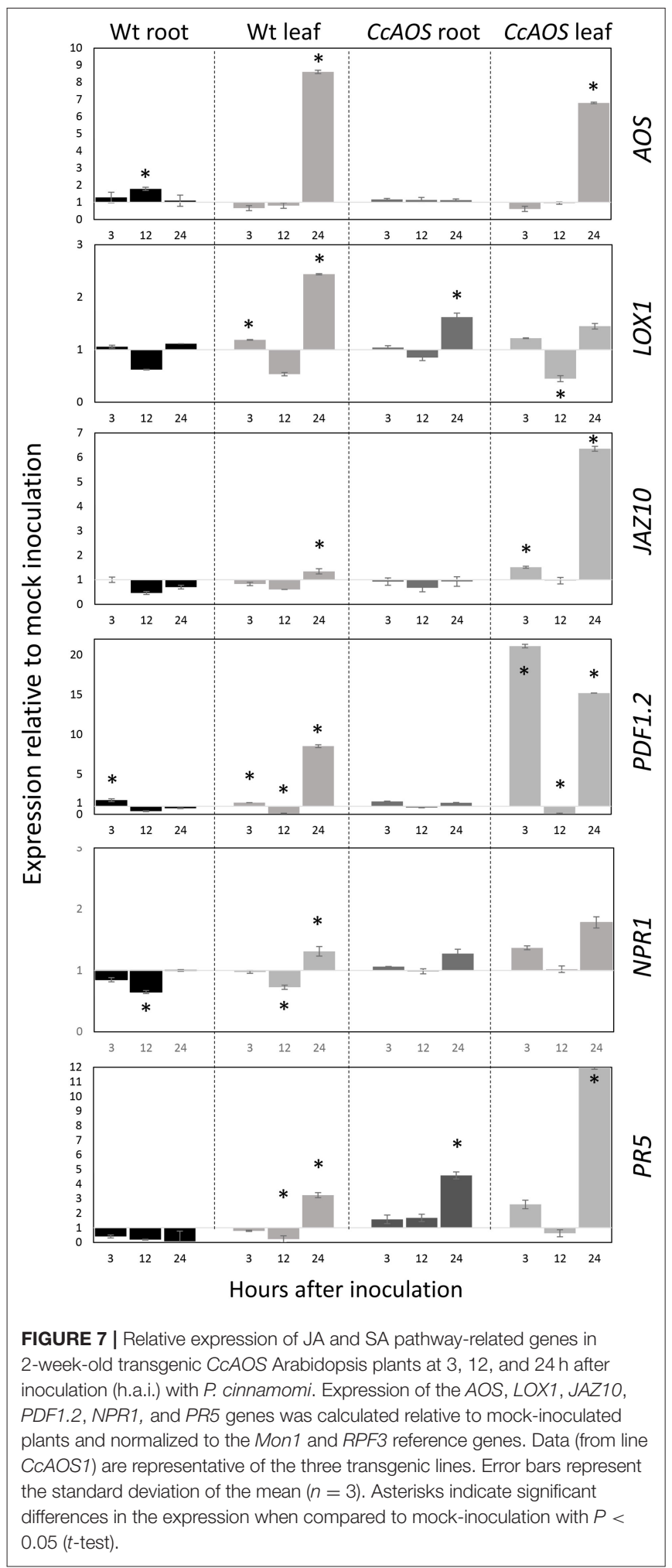

siliques (Figure 8, fertile plants). Upon genotyping, the nondisrupted AtAOS allele from the original transgenic pollen was detected in these plants along with the CCAOS transgene and
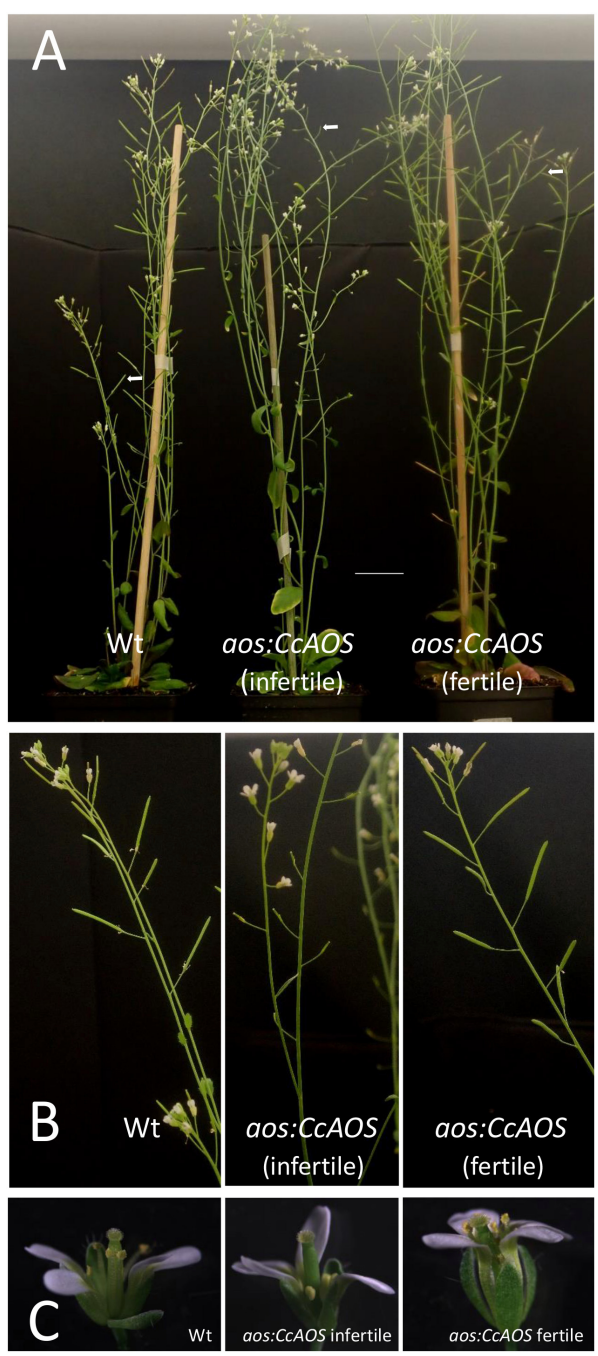

FIGURE 8 | Genetic crossing of a CCAOS transgenic line with the Arabidopsis aos-GK624b02 mutant. (A) Two-month-old Col-0 wild type, aos:CCAOS infertile (no inherited AtAOS) and aos:CcAOS fertile (inherited AtAOS) F2 plants. Arrows indicate siliques. Scale bar $=3 \mathrm{~cm}$. (B) Detail of flower stalks with siliques. (C) Detail of flowers.

the T-DNA disrupted AtAOS (Supplementary Figure 4). The remaining $6 \mathrm{~F} 2$ plants produced small anthers with scarce pollen and abnormal siliques (Figure 8, infertile plants). Genotyping of these plants detected the presence of CcAOS and the disrupted AtAOS, but no wild type AtAOS gene (Supplementary Figure 4). Thus, heterologous expression of the C. crenata AOS gene was unable to restore fertility of the Arabidopsis aos insertion mutant, suggesting a distinct biological function from the endogenous AtAOS.

To evaluate whether the A.thaliana AOS is involved in P. cinnamomi resistance and confirm a role for CcAOS in defense responses, we compared mycelia progression in wild type, aosGK624b02 mutant and aosGK624b02:CcAOS F3 plants upon inoculation with P. cinnamomi (Figure 9). After germination 


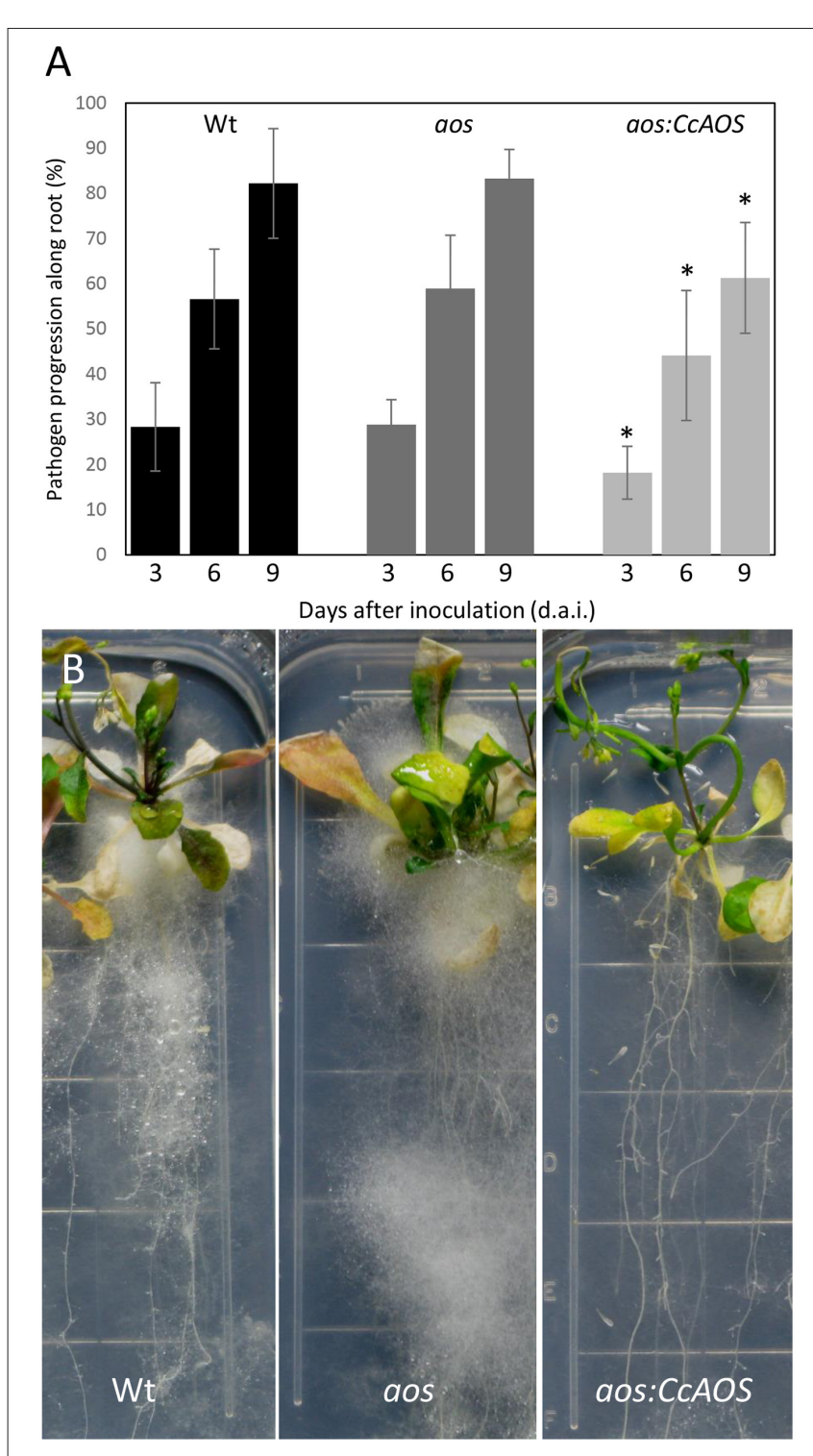

FIGURE 9 | Phytophthora cinnamomi progression in wild type (Wt), aos-GK624b02 mutant (aos), and CCAOS-expressing plants. (A) Percentage of $P$. cinnamomi progression along roots 3,6 , and 9 days after inoculation (d.a.i.). Asterisks refers to significant differences from the Col-0 wild type at each time-point $(P<0.001, t$-test). Error bars represent the standard deviation of the mean $(n=3)$. (B) Mycelia accumulation along roots and around the plant's aerial part, 3 weeks after inoculation. Grid squares: $1.4 \mathrm{~cm} /$ side.

in selection media, 2-week-old plants were inoculated and data collected during 9 days. No statistically significant differences were observed between wild type and aosGK624b02 plants (Figure 9A) indicating that the endogenous AtAOS does not play a role in resistance to the oomycetes. However, while a clear accumulation of mycelia was observed in roots and aerial tissues of the Col-0 wild type and the aos-GK624b02 mutant, aosGK624b02:CcAOS plants exhibited a marked delay in pathogen advance on roots (Figure 9B), clearly substantiating the notion that $C$. crenata AOS confers resistance to $P$. cinnamomi in A. thaliana.

\section{DISCUSSION}

We previously proposed an important role for allene oxide synthase (AOS) in the defense mechanisms of the Japanese chestnut, Castanea crenata, against the pathogenic oomycete Phytophthora cinnamomi (Serrazina et al., 2015). To further investigate and confirm the significance of the C. crenata AOS in this biotic stress response, we resorted to its heterologous expression and functional characterization using an Arabidopsis Ler-0 ecotype- $P$. cinnamomi pathosystem. To assess correct localization of the expressed CcAOS protein, its sequence was fused with the eGFP reporter gene and fluorescence followed both in transgenic Arabidopsis plants and upon transient expression in $N$. benthamiana leaves. Furthermore, we used a loss-of-function allele for the endogenous $A$. thaliana AOS gene and its genetic crossing with the transgenic CcAOS Arabidopsis line to confirm a role for the chestnut AOS protein in pathogen defense and gain insight into the function of the AOS enzyme in the two plant species.

\section{CcAOS Confers Oomycete Resistance to Arabidopsis Without Compromising Plant Growth and Fertility}

Like the European chestnut Castanea sativa, Arabidopsis plants of the Ler-0 ecotype are susceptible to P. cinnamomi (Robinson and Cahill, 2003). Given the amenability to transformation and functional characterization studies, we used this model flowering plant to express the Castanea crenata AOS gene, which we previously found to be upregulated in this species upon oomycete infection (Serrazina et al., 2015). Using the strong constitutive $35 \mathrm{~S}$ promoter, CCAOS was constitutively expressed in Arabidopsis, as assessed in three independent transformed plant lines by fluorescence confocal microscopy and western blotting.

Our results show that, morphologically, transgenic CcAOS plants display mild differences when compared to wild type. Arabidopsis plants expressing the CcAOS transcript are distinguished by its higher growth rate, smaller siliques and lower number of seeds when compared with their WT counterparts. As previously reported by Farmer and Goossens (2019), higher basal level of JA due to an upregulation of CcAOS, resulted in plants with higher growth rate, a mechanism that could also be occurring in our heterologous lines. Interestingly, Kubigsteltig and Weiler (2003) obtained several Arabidopsis lines affected in the transcriptional control of AOS and, from eight lines showing constitutive AOS expression, all showed signs of growth inhibition, suggesting a different role for CcAOS which may account for the resistance exhibited by $C$. crenata. In two of those lines, flower development and anther size were so affected that they failed to set seed (Kubigsteltig and Weiler, 2003). In the present study, transgenic CcAOS plants were still able to generate viable seed set, though Kubigsteltig and Weiler (2003) did not present results on the fertilization level of the other classes of fertile mutants that would enable a comparison with CcAOS plants. Regardless that in all CcAOS plants a correlation was not evident between transcripts levels and protein expression (or with pathogen progress/quantity), in 
all lines isolated the pathogen progression was delayed, and the amounts of pathogen DNA were reduced when compared to non-transformed plants. Our data therefore provides compelling evidence that the $C$. crenata $A O S$ gene positively regulates plant tolerance to oomycete infection in Arabidopsis.

The implemented Arabidopsis - P. cinnamomi pathosystem in axenic conditions provided us with a controlled and confined system which allows both the following of the pathogen progression and plant adaptation throughout the time. We focused on the first 5 d.a.i. for microscopic observations of root and pathogen tissues and on 12 d.a.i. for mycelia progression along the root system. After these time periods a substantial invasion of the mycelia over and inside root tissues hampered further clear observations. Despite the massive hyphal invasion, neither wild type or transgenic CCAOS plants showed necrotic tissues in roots. Arabidopsis ecotypes less susceptible to P. cinnamomi, such as Col-0, promote the formation of callose plugs in root infected areas, as well as hypersensitive response and necrotic lesions in leaves, in order to restrain the pathogen (Robinson and Cahill, 2003; Rookes et al., 2008). Here, Ler-0 root staining with aniline blue did not reveal distinct production of callose plugs (not shown), in agreement with the susceptibility phenotype described for this ecotype (Robinson and Cahill, 2003). Similar results were observed for CcAOS roots. Notably, the transgenic CcAOS plants generated in the Ler-0 background exhibited a significant reduction in mycelia progression along roots and in $P$. cinnamomi DNA levels, demonstrating a notable contribution of the C. crenata AOS to plant defense.

\section{CcAOS Role Involves Changes in the Jasmonic and Salicylic Acid Pathways}

Our results on the expression of endogenous AOS in inoculated Ler-0 wild type plants agree with a susceptibility phenotype - virtually unaffected in roots and significantly upregulated in leaves $24 \mathrm{~h}$ after inoculation (h.a.i.). This is consistent with reports that AOS activity is highly enhanced in Arabidopsis leaves after wounding (Laudert et al., 2000). Our observation that endogenous AOS expression follows a similar pattern in transgenic CCAOS plants supports a non-deleterious effect of the heterologous protein in the morphology and development of transformed plant lines. Taken together, our data indicate that the lower susceptibility of transgenic CcAOS plants to the oomycete $P$. cinammomi results specifically from the introduction (and expression) of the CcAOS gene and its downstream effects.

JA and SA are two of the main phytohormones implicated in plant responses against pathogens (Clarke et al., 2000; Rookes et al., 2008). Most of the studies performed so far were conducted in leaves and reported an antagonistic interaction between JA and SA signaling (Chuberre et al., 2018). However, Attard et al. (2010) reported that in roots of Arabidopsis thaliana both phytohormones are activated coordinately upon Phytophthora parasitica infection, suggesting that the early defense activation differs between roots and leaves. In the present study, we observed significant changes in the transcript levels of JA- and SA- marker genes in Arabidopsis Ler-0 inoculated with $P$. cinnamomi. Lower expression levels were recorded in roots in agreement with Rookes et al. (2008) and Attard et al. (2010); the root was the site of pathogen inoculation and Ler0 plants present the most susceptible background, allowing the pathogen to efficiently suppress host immunity. The expression of LOX1, AOS, JAZ10, PDF1.2 (JA-related), and PR5 (SA-related) showed relevant upregulation in leaves at 24 h.a.i., revealing an amplification of the response far from the sites of inoculation (roots). Once a response to the pathogen is triggered, roots can induce a response in non-challenged organs (such as leaves), corresponding to a systemic acquired resistance (SAR, reviewed by Chuberre et al., 2018).

In roots of transgenic CcAOS plants, upregulation of $L O X 1$ expression at 24 h.a.i. was higher than in the wild type counterpart. This agrees with our predicted role of CcAOS in the response to biotic stress since LOX1 acts in the systemic defense against bacterial pathogens in Arabidopsis roots as shown by Vicente et al. (2012). In this report, the authors suggested that LOX1 mediates the production of oxilipins of the 9-lipoxygenase pathway which, in turn, activate SAR and regulate lateral root development (Vicente et al., 2012). In Arabidopsis roots, 9oxilipins may activate cell wall-based responses to the fungus Golovinomyces cichoracearum (Marcos et al., 2015). A causal relationship between the constitutive expression of CcAOS and the higher levels of LOX1 in roots cannot be unequivocally established; LOX1 is in the 9-lipoxygenase pathway and AOS is in the 13-lipoxygenase pathway. However, a role for oxylipins in the adaptation to adverse growth conditions and defense responses is well-established (Armengaud et al., 2004) and their biosynthesis is initiated by the action of 9-LOX and 13-LOX (Vellosillo et al., 2007). Thus, a higher metabolism of common polyunsaturated fatty acids may stimulate both pathways. Moreover, LOX proteins expressed upon apple climacteric ripening were found to have dual (9/13) positioning specific lipoxygenase function (Schiller et al., 2015).

JAZ10 upregulation in leaves of transgenic CCAOS plants 24 h.a.i. was also clear and in contrast with the absence of differential regulation in roots. Yan et al. (2007) suggested that JAZ10 is responsible for a repression of JA-regulated growth retardation in wounded roots of the Arabidopsis Col-0 ecotype. However, the absence of differential regulation of JAZ10 in roots upon $P$. cinnamomi inoculation suggests an inhibitory effect of the pathogen on the defense response in its site of action. In leaves, JAZ10 expression showed opposite regulation, in phase with the upregulation of $A O S$ at the same time-point (24 h.a.i.). Leaves are directly exposed to light and, under such conditions, the JA pathway is highly inducible, with a higher expression of AOS that leads to biologically active jasmonoyl-isoleucine (JAIle) production (Farmer and Goossens, 2019). The availability of JA-Ile stimulates the fine modulation of the JA signaling (e.g., by JAZ10) which could promote growth and recovery from an infection scenario, giving the plant a chance of reproduction (Guo et al., 2018; Farmer and Goossens, 2019). JAZ proteins, through immunity-repression of JA pathway, promote growth to limit carbon starvation associated with strong defense responses and enable reproduction (Guo et al., 2018). 
Among the analyzed genes, PDF1.2 showed the highest up-regulation in leaves of transgenic CcAOS plants 3 h.a.i., suggesting that CcAOS promotes the synthesis of JA and consequently the expression of the JA-responsive gene PDF1.2. The regulation pattern corroborates the abundance of PDF1.2 transcript 2.5 h.a.i. in leaves infected with $P$. parasitica (Attard et al., 2010). The noticeable upregulation of PDF1.2 at 3 h.a.i. contrasts with the modest upregulation in wild type leaves, pointing to a relevant role of the plant defensin PDF1.2 in the defense response of CcAOS plants to the pathogen.

NPR1 and PR5 have been used as markers for SA signaling and SAR (revised in Sticher et al., 1997). NPR1 is associated to SAR as a negative regulator of the JA pathway (Derksen et al., 2013). In our study, the upregulation of PR5 in wild type and transgenic CcAOS leaves was preceded by a weak induction of NPR1, suggesting that the crosstalk between JA and SA pathways is not relevant at the NPR1 level. Similarly, Eshragui et al. (2011a) reported that the application of phosphite (a systemic chemical elicitor of defense responses to $P$. cinnamomi) to Ler-0 leaves, induced the expression of PR5, but not of NPR1. Our results corroborate such report and suggest a cooperation between the SA and JA pathways in the defense response independent of NPR1. Previously, Clarke et al. (2000) described a SA-mediated NPR1-independent resistance response that requires JA and ethylene signaling, in Arabidopsis challenged with the oomycete Perenospora parasitica. The most significant upregulation of PR5 24 h.a.i. in leaves and roots of transgenic CcAOS plants points to a relevant role of SA signaling in the defense response to $P$. cinnamomi. The induction of $P R 5$ in CCAOS roots, in opposition to the overall downregulation in wild type roots, indicates that the heterologous expression of CcAOS affects the regulation of the SA pathway to improve the early steps of the defense response to the pathogen.

\section{CcAOS Traffics to Plastids and Fulfills a Different Biological Function From AtAOS}

The viability of heterologous expression of CcAOS as a tool to engineer cultivars less susceptible to pathogen attack requires confirmation that the protein is functional in pathogen defense and, inherently, that it localizes to the correct sub-cellular compartment to perform its catabolic activity. Here we addressed these two issues through expression of the C. crenata AOS in different $A$. thaliana backgrounds and live imaging of a CcAOSeGFP fusion protein both in transgenic Arabidopsis plants and in $N$. benthamiana leaf epidermal cells.

Concerning the protein localization, we observed that an eGFP-CcAOS fusion protein was targeted to the expected final destination, the plastid. However, and quite interestingly, the observations performed in leaf epidermal cells of $N$. benthamiana showed trafficking through reticulate-like structures. It is tempting to speculate that the lower oomycete susceptibility induced by CcAOS results from post-translational processing of the protein through the endomembrane compartment. Endoplasmic reticulum (ER)-bodies accumulate defense proteins (Chuberre et al., 2018) and the ER is now recognizably a hub to sort proteins to plastids and mitochondria (Bellucci et al.,
2018). Activation of endomembrane trafficking associated to an increase in salicylic acid levels during plant defense has also been reported (Ruano and Scheuring, 2020). Although appealing, this hypothesis clearly requires further experimental evidence.

Most significantly, when compared to the wild type plants, the expression of CCAOS in the aos mutant line resulted in a beneficial effect upon $P$. cinnamomi attack. This suggests that even in an aos-JA deficient background, CcAOS is sufficient to boost pathogen defense. The absence of significant differences in pathogen progression between the wild type and a newly isolated aos knockout, for which we confirmed previous reports of reduced fertility (Park et al., 2002; von Malek et al., 2002), indicates that the endogenous AtAOS does not play a significant role in the defense to $P$. cinnamomi. In support of this observation, Rookes et al. (2008) did not find differences between wild type Col-0 and the JA-biosynthesis mutants coil and jar1 upon P. cinnamomi root inoculation. Nonetheless, genetic crossing of transgenic CcAOS plants with the aos mutant also resulted in a beneficial effect upon $P$. cinnamomi attack, corroborating a role for the chestnut enzyme in the defense response to the pathogen. Noteworthily, expression of CCAOS in the male sterile aos mutant background led to plants that were morphologically similar to the wild type but still infertile. This indicates that, although functionally active in Arabidopsis, CcAOS fulfills distinct functions from the endogenous Arabidopsis AOS.

Taken together, the data presented here support the notion that CcAOS promotes resistance of the Japanese chestnut to oomycete pathogens and that its constitutive expression could be a valid tool to engineer cultivars from other species to overcome susceptibility (e.g., C. sativa) (Santos et al., 2017). Moreover, accordingly to Camisón et al. (2019), the expression of CcAOS seems to induce the jasmonic and salycilic acid pathways (upregulation of $L O X 1, J A Z 10$, and PR5) contributing to a more efficient host response in the initial stages of P. cinnamomi infection without compromising growth and fertility. It further raises new questions about the evolution of plant lipid regulation and how protein function is achieved beyond catalytic activity.

It should be noted that the basis of the resistance to P. cinnamomi is multigenic (López-Villamor et al., 2018) and that the CcAOS gene by itself does not fully revert the susceptibility phenotype of Ler-0. In fact, CcAOS plants did not show hypersensitive response or the characteristic production of callose plugs (Robinson and Cahill, 2003). Nonetheless, the signs of enhanced systemic acquired resistance from roots to leaves (suggested by the upregulation of PR5 in inoculated CCAOS roots) point to a priming of defense responses in surrounding plants through the volatile compounds from JA- and SA-pathways (Chuberre et al., 2018).

\section{DATA AVAILABILITY STATEMENT}

The original contributions presented in the study are included in the article/Supplementary Materials, further inquiries can be directed to the corresponding authors. 


\section{AUTHOR CONTRIBUTIONS}

SS and RM conceived and planned the experiments and wrote the manuscript. HM prepared the pathogen. RC planned the experiments. PD analyzed the data and wrote the manuscript. SS performed the experiments. All authors contributed to the article and approved the submitted version.

\section{FUNDING}

This work was supported by Fundação para a Ciência e a Tecnologia (FCT/MCTES/PIDDAC, Portugal) through postdoctoral fellowship SFRH/BPD/108653/2015 and the work contract 2477 of the contract-program 12345/20181 defined by DL nr. 57/2016 awarded to SS, as well as through research funds awarded to RM (UIDB/04046/2020 and UIDP/04046/2020) and PD (UIDB/04551/2020).

\section{REFERENCES}

Armengaud, P., Breitling, R., and Amtmann, A. (2004). The potassium-dependent transcriptome of Arabidopsis reveals a prominent role of jasmonic acid in nutrient signaling. Plant Physiol. 136, 2556-2576. doi: 10.1104/pp.104.0 46482

Attard, A., Gourgues, M., Callemeyn-Torre, N., and Keller, H. (2010). The immediate activation of defense responses in Arabidopsis roots is not sufficient to prevent Phytophthora parasitica infection. New Phytol. 187, 449-460. doi: 10.1111/j.1469-8137.2010.03272.x

Balfagón, D., Sengupta, S., Gómez-Cadenas, A., Fritschi, F. B., Azad, R. K., Mittler, R., et al. (2019). Jasmonic acid is required for plant acclimation to a combination of high light and heat stress. Plant Physiol. 181, 1668-1682. doi: 10.1104/pp.19.00956

Bellucci, M., De Marchis, F., and Pompa, A. (2018). The endoplasmic reticulum is a hub to sort proteins toward unconventional traffic pathways and endosymbiotic organelles. J. Exptl. Bot. 69, 7-20. doi: 10.1093/jxb/erx286

Betsuyaku, S., Katou, S., Takebayashi, Y., Sakakibara, H., Nomura, N., and Fukuda, H. (2018). Salicylic acid and jasmonic acid pathways are activated in spatially different domains around the infection site during effector-triggered immunity in Arabidopsis thaliana. Plant Cell Physiol. 59, 8-16. doi: 10.1093/pcp/pcx181

Camisón, Á., Martín, M. Á., Sánchez-Bel, P., Flors, V., Alcaide, F., Morcuende, D., et al. (2019). Hormone and secondary metabolite profiling in chestnut during susceptible and resistant interactions with Phytophthora cinnamomi. J. Plant Physiol. 241:153030. doi: 10.1016/j.jplph.2019.1 53030

Chapple, C. (1998). Molecular-genetic analysis of plant cytochrome P450dependent monooxygenases. Ann. Rev. Plant Physiol. Plant Mol. Biol. 49, 311-343. doi: 10.1146/annurev.arplant.49.1.311

Chen, Y. C., Wong, C. L., Muzzi, F., Vlaardingerbroek, I., Kidd, B. N., and Schenk, P. M. (2015). Root defense analysis against Fusarium oxysporum reveals new regulators to confer resistance. Sci. Rep. 4:5584. doi: 10.1038/srep05584

Chuberre, C., Plancot, B., Driouich, A., Moore, J. P., Bardor, M., Bruno, G., et al. (2018). Plant immunity is compartmentalized and specialized in roots. Frontiers Pl. Sci. 9:1692. doi: 10.3389/fpls.2018.01692

Clarke, J. D., Volko, S. M., Ledford, H., Ausubel, F. M., and Dong, X. (2000). Roles of salicylic acid, jasmonic acid, and ethylene in $c p r$-induced resistance in Arabidopsis. Plant Cell 12, 2175-2190. doi: 10.1105/tpc.12.11.2175

Czechowski, T., Stitt, M., Altmann, T., Udvardi, M. K., and Scheible, W.R. (2005). Genome-Wide identification and testing of superior reference genes for transcript normalization in Arabidopsis. Plant Physiol. 139, 5-17. doi: $10.1104 /$ pp. 105.063743

\section{ACKNOWLEDGMENTS}

The authors are grateful to N.-H- Chua for the pBA-eGFP binary vector, $V$. Nunes (IGC) for supervising and assistance with Arabidopsis transformation, E. Novo-Uzal (IGC) for supervising and assistance with western blotting, A.B. da Silva (FCUL) for advising on Arabidopsis phenotyping, T. Braga (FCUL) for assistance with $N$. benthamiana imaging and A. M. Fortes (FCUL) for critical reviewing of the manuscript. Imaging was performed at the Faculty of Sciences of the University of Lisbon's Microscopy Facility, a node of the Portuguese Platform for BioImaging (PPBI-POCI-01-0145-FEDER-022122).

\section{SUPPLEMENTARY MATERIAL}

The Supplementary Material for this article can be found online at: https://www.frontiersin.org/articles/10.3389/fpls.2021. 628697/full\#supplementary-material

De Coninck, B., Timmermans, P., Vos, C., Cammue, B. P. A., and Kazan, K. (2014). What lies beneath: belowground defense strategies in plants. Trends Pl. Sci. 20, 91-101. doi: 10.1016/j.tplants.2014.09.007

Derksen, H., Rampitsch, C., and Daayf, F. (2013). Signaling cross-talk in plant disease resistance. Plant Sci. 207, 79-87. doi: 10.1016/j.plantsci.2013.03.004

Devoto, A., and Turner, J. G. (2005). Jasmonate-regulated Arabidopsis stress signalling network. Physiol. Plantarum 123, 161-172. doi: 10.1111/j.1399-3054.2004.00418.x

Dumin, W., Rostás, M., and Winefield, C. (2018). Identification and functional characterisation of an allene oxide synthase from grapevine (Vitis vinifera L. Sauvignon blanc). Mol. Biol. Rep. 45, 263-277. doi: 10.1007/s11033-018-4159-y

Eshragui, L., Anderson, J. P., Aryamanesh, N., Shearer, B., McComb, J. A., Hardy, G. E. S. J., et al. (2011a). Phosphite primed defence responses and enhanced expression of defence genes in Arabidopsis thaliana infected with Phytophthora cinnamomi. Plant Pathol. 60, 1086-1095. doi: 10.1111/j.1365-3059.2011.02471.x

Eshragui, L., Aryamanesh, N., Anderson, J. P., Shearer, B., McComb, J. A., Hardy, G. E. S. J., et al. (2011b). A quantitative PCR assay for accurate in planta quantification of the necrotrophic pathogen Phytophthora cinnamomi. Eur. J. Plant Pathol. 131, 419-430. doi: 10.1007/s10658-011-9819-x

Farmer, E. E., and Goossens, A. (2019). Jasmonates: what ALLENE OXIDE SYNTHASE does for plants. J. Exptl. Bot. 70, 3373-3378. doi: 10.1093/jxb/erz254

Froehlich, J. A., Itoh, A., and Howe, G. A. (2001). Tomato Allene Oxide Synthase and fatty acid hydroperoxide lyase, two Cytochrome P450s involved in oxylipin metabolism, are targeted to different membranes of chloroplast envelope. Plant Physiol. 125, 306-317. doi: 10.1104/pp.125.1.306

Guo, Q., Yoshida, Y., Major, I. T., Wang, K., Sugimoto, K., Kapali, G., et al. (2018). JAZ repressors of metabolic defense promote growth and reproductive fitness in Arabidopsis. Proc. Natl. Acad. Sci. U. S. A. 115, E10768-E10777. doi: 10.1073/pnas.1811828115

Herlihy, J., Ludwig, N. R., van den Ackerveken, G., and McDowell, J. M. (2019). Oomycetes used in Arabidopsis research. Arabidopsis Book 17, 1-26. doi: $10.1199 /$ tab.0188

Kleinboelting, N., Huep, G., Kloetgen, A., Viehoever, P., and Weisshaar, B. (2012). GABI-Kat SimpleSearch: new features of the Arabidopsis thaliana T-DNA mutant database. Nucleic Acids Res. 40, D1211-D1215. doi: 10.1093/nar/gkr1047

Kubigsteltig, I. I., and Weiler, E. W. (2003). Arabidopsis mutants affected in the transcriptional control of allene oxide synthase, the enzyme catalyzing the entrance step in octadecanoid biosynthesis. Planta 217, 748-757. doi: $10.1007 / \mathrm{s} 00425-003-1056-\mathrm{x}$ 
Laudert, D., Schaller, F., and Weiler, E. W. (2000). Transgenic Nicotiana tabacum and Arabidopsis thaliana plants overexpressing allene oxide synthase. Planta 211, 163-165. doi: 10.1007/s004250000316

Laudert, D., and Weiler, E. W. (1998). Allene oxide synthase: a major control point in Arabidopsis thaliana octadecanoid signalling. Plant J. 15, 675-684. doi: 10.1046/j.1365-313x.1998.00245.x

López-Villamor, A., Fernández-López, J., Míguez-Soto, B., and Sánchez, M. E. (2018). Resistance to Phytophthora cinnamomi in Castanea spp. is under moderately high genetic control mainly because of additive genetic variance. Euphytica 214:230. doi: 10.1007/s10681-018-2309-x

Marcos, R., Izquierdo, Y., Vellosillo, T., Kulasekaran, S., Cascón,. T., Hamberg, M., et al. (2015). 9-Lipoxygenase-derived oxylipins activate brassinosteroid signaling to promote cell wall-based defense and limit pathogen infection. Plant Physiol. 169, 2324-2334. doi: 10.1104/pp.15.00992

Martinez Trujillo, M., Limones-Briones, V., Cabrera-Ponce, J. L., and HerreraEstrella, L. (2004). Improving transformation efficiency of Arabidopsis thaliana by modifying the floral dip method. Plant Mol. Biol. Reporter 22, 63-70. doi: 10.1007/BF02773350

Maurel, M., Robin,. C., Simonneau, T., Loustau, D., Dreyer, E., and DesprezLoustau, M.-L. (2004). Stomatal conductance and root-to-shoot signalling in chestnut saplings exposed to Phytophthora cinnamomi or partial soil drying. Funct. Plant Biol. 31, 41-51. doi: 10.1071/FP03133

Moralejo, E., García-Muñoz, J. A., and Descals, E. (2009). Susceptibility of Iberian trees to Phytophthora ramorum and P. Cinnamomi. Plant Pathol. 58, 271-283. doi: 10.1111/j.1365-3059.2008.01956.x

Murashige, T., and Skoog, F. (1962). A revised medium for rapid growth and bioassays with tobacco tissue cultures. Physiol. Plant. 15, 473-497. doi: 10.1111/j.1399-3054.1962.tb08052.x

Pajerowska-Mukhtar, K. M., Mukhtar, M. S., Guex, N., Halim, V. A., Rosahl, S., Somssich, I. E., et al. (2008). Natural variation of potato allene oxide synthase 2 causes differential levels of jasmonates and pathogen resistance in Arabidopsis. Planta 228, 293-306. doi: 10.1007/s00425-008-0737-x

Park, J.-H., Halitschke, R., Kim, H. B., Baldwin, I. T., Feldmann, K. A., and Feyereisen, R. (2002). A knock-out mutation in allene oxide synthase results in male sterility and defective wound signal transduction in Arabidopsis due to a block in jasmonic acid biosynthesis. Plant J. 31, 1-12. doi: 10.1046/j.1365-313X.2002.01328.x

Peng, Q., Zhou, Y., Liao, Y., Zeng, L., Xu, X., Jia, Y., et al. (2018). Functional characterization of an Allene Oxide Synthase involved in biosynthesis of jasmonic acid and its influence on metabolite profiles and ethylene formation in tea (Camellia sinensis) flowers. Intl. J. Mol. Sci. 19:2440. doi: 10.3390/ijms19082440

Proietti, S., Caarls, L., Coolen, S., Van Pelt, J. A., Van Wees, S. C. M., and Pieterse, C. M. J. (2018). Genome-wide association study reveals novel players in defense hormone crosstalk in Arabidopsis. Plant Cell Environm. 41, 2342-2356. doi: 10.1111/pce.13357

Redondo, M. A., Pérez-Sierra, A., Abad-Campos, P., Torres, L., Solla, A., Reig-Armiñana, J., et al. (2015). Histology of Quercus ilex roots during infection by Phytophthora cinnamomi. Trees 29, 1943-1957. doi: 10.1007/s00468-015-1275-3

Robinson, L. H., and Cahill, D. M. (2003). Ecotypic variation in the response of Arabidopsis thaliana to Phytophthora cinnamomi. Aust. Plant Pathol. 32, 53-64. doi: 10.1071/AP02064

Rookes, J. E., Wright, M. L., and Cahill, D. M. (2008). Elucidation of defence responses and signalling pathways induced in Arabidopsis thaliana following challenge with Phytophthora cinnamomi. Physiol. Mol. Plant Pathol. 72, 151-161. doi: 10.1016/j.pmpp.2008.08.005

Ruano, G., and Scheuring, D. (2020). Plant cells under attack: unconventional endomembrane trafficking during plant defense. Plants 9:389. doi: 10.3390/plants9030389

Santos, C., Duarte, S., Tedesco, S., Fevereiro, P., and Costa, R. (2017). Expression profiling of Castanea genes during resistant and susceptible interactions with the oomycete pathogen Phytophthora cinnamomi reveal possible mechanisms of immunity. Frontiers Pl. Sci. 8:515. doi: 10.3389/fpls.2017.00515

Schiller, D., Contreras, C., Vogt, J., and Schwab, W. (2015). A dual positional specific lipoxygenase functions in the generation of flavor compounds during climacteric ripening of apple. Horticulture Res. 2:15003. doi: 10.1038/hortres.2015.3

Schlaeppi, K., Abou-Mansour, E., Buchala, A., and Mauch, F. (2010). Disease resistance of Arabidopsis to Phytophthora brassicae is established by the sequential action of indole glucosinolates and camalexin. Plant J. 62, 840-851. doi: 10.1111/j.1365-313X.2010.04197.x

Schmittgen, T. D., and Livak, K. J. (2008). Analyzing real-time PCR data by the comparative $\mathrm{C}_{\mathrm{T}}$ method. Nat. Protoc. 3, 1101-1108. doi: 10.1038/nprot.2008.73

Scholz, J., Brodhun, F., Hornung, E., Herrfurth, C., Stumpe, M., Beike, A. K., et al. (2012). Biosynthesis of allene oxides in Physcomitrella patens. BMC Plant Biol. 12:228. doi: 10.1186/1471-2229-12-228

Serrazina, S., Santos, C., Machado, H., Pesquita, C., Vicentini, R., Pais, M. S., et al. (2015). Castanea root transcriptome in response to Phytophthora cinnamomi challenge. Tree Gen. Genomes 11:6. doi: 10.1007/s11295-014-0829-7

Sparkes, I. A., Runions, J., Kearns, A., and Hawes, C. (2006). Rapid, transient expression of fluorescent fusion proteins in tobacco plants and generation of stably transformed plants. Nat. Protoc. 1, 2019-2025. doi: 10.1038/nprot.2006.286

Sperschneider, J., Catanzariti, A. M., DeBoer, K., Petre, B., Gardiner, D. M., Singh, K. B., et al. (2017). LOCALIZER: subcellular localization prediction of both plant and effector proteins in the plant cell. Sci. Rep. 7:44598. doi: $10.1038 /$ srep44598

Sticher, L., Mauch-Mani, B., and Métraux, J. P. (1997). Systemic acquired resistance. Ann. Rev. Phytopathol. 35, 235-270. doi: 10.1146/annurev.phyto.35.1.235

Tijet, N., and Brash, A. R. (2002). Allene oxide synthases and allene oxides. Prostaglandins and Other Lipid Mediat. 68-69, 423-431. doi: 10.1016/s0090-6980(02)00046-1

Vellosillo, T., Martínez, M., López, M. A., Vicente, J., Cascón, T., Dolan, L., et al. (2007). Oxylipins produced by the 9-Lipoxygenase Pathway in Arabidopsis regulate lateral root development and defense responses through a specific signaling cascade. Plant Cell 19, 831-846. doi: 10.1105/tpc.106.046052

Vicente, J., Cascón, T., Vicedo, B., García-Agustín, P., Hamberg, M., and Castresana, C. (2012). Role of 9-lipoxygenase and $\alpha$-dioxygenase oxylipin pathways as modulators of local and systemic defense. Mol. Plant 5, 914-928. doi: $10.1093 / \mathrm{mp} / \mathrm{ssr} 105$

von Malek, B., van der Graaff, E., Schneitz, K., and Keller, B. (2002). The Arabidopsis male-sterile mutant dde2-2 is defective in the ALLENE OXIDE SYNTHASE gene encoding one of the key enzymes of the jasmonic acid biosynthesis pathway. Planta 216, 187-192. doi: 10.1007/s00425-002-0906-2

Willetts, H. J. (1997). Morphology, development and evolution of stromata/sclerotia and macroconidia of the Sclerotiniaceae. Mycological Res. 101, 939-952. doi: 10.1017/S0953756297003559

Yan, Y., Stolz, S., Chételat, A., Reymond, P., Pagni, M., Dubugnon, L., et al. (2007). A downstream mediator in the growth repression limb of the jasmonate pathway. Plant Cell 19, 2470-2483. doi: 10.1105/tpc.107.050708

Conflict of Interest: The authors declare that the research was conducted in the absence of any commercial or financial relationships that could be construed as a potential conflict of interest.

Copyright (c) 2021 Serrazina, Machado, Costa, Duque and Malhó. This is an openaccess article distributed under the terms of the Creative Commons Attribution License (CC BY). The use, distribution or reproduction in other forums is permitted, provided the original author(s) and the copyright owner(s) are credited and that the original publication in this journal is cited, in accordance with accepted academic practice. No use, distribution or reproduction is permitted which does not comply with these terms. 